\title{
String graphs and separators
}

\author{
JiŘí MATOUŠEK* \\ Department of Applied Mathematics \\ Charles University, Malostranské nám. 25 \\ 11800 Praha 1, Czech Republic, and \\ Institute of Theoretical Computer Science \\ ETH Zurich, 8092 Zurich, Switzerland
}

\begin{abstract}
String graphs, that is, intersection graphs of curves in the plane, have been studied since the 1960s. We provide an expository presentation of several results, including very recent ones: some string graphs require an exponential number of crossings in every string representation; exponential number is always sufficient; string graphs have small separators; and the current best bound on the crossing number of a graph in terms of paircrossing number. For the existence of small separators, the proof includes generally useful results on approximate flow-cut dualities.
\end{abstract}

This expository paper was prepared as a material for two courses co-taught by the author in 2013, at Charles University and at ETH Zurich. It aims at a complete and streamlined presentation of several results concerning string graphs. This important and challenging class of intersection graphs has traditionally been studied at the Department of Applied Mathematics of the Charles University, especially by Jan Kratochvíl and his students and collaborators.

A major part of the paper is devoted to a separator theorem by Fox and Pach, recently improved by the author, as well as an application of it by Tóth in a challenging problem from graph drawing, namely, bounding the crossing number by a function of the pair-crossing number. This is an excellent example of a mathematical proof with a simple idea but relying on a number of other results from different areas. The proof is presented in full, assuming very little as a foundation, so that the reader can see everything that is involved. A key step is an approximate flow-cut duality from combinatorial optimization and approximation algorithms, whose proof relies on linear programming duality and a theorem on metric embeddings.

Acknowledgments. I am very grateful to Rado Fulek, Vincent Kusters, Jan Kynčl, and Zuzana Safernová for proofreading, comments, and corrections. It was a pleasure to teach the courses together with Pavel Valtr in Prague and with Michael Hoffmann and Emo Welzl in Zurich, and to work with Jan Kratochvíl on questions in string graphs as well as on many other things. I also thank an anonymous referee for numerous useful remarks and suggestions.

*Supported by the ERC Advanced Grant No. 267165 and by the project CE-ITI (GACR P202/12/G061). 


\section{$1 \quad$ Intersection graphs}

The classes $\operatorname{IG}(\mathcal{M})$. Let $\mathcal{M}$ be a system of sets; we will typically consider systems of geometrically defined subsets of $\mathbb{R}^{2}$, such as all segments in the plane. We define $\operatorname{IG}(\mathcal{M})$, the class of intersection graphs of $\mathcal{M}$, by

$$
\operatorname{IG}(\mathcal{M})=\left\{(V, E): V \subseteq \mathcal{M}, E=\left\{\left\{M, M^{\prime}\right\} \in\left(\begin{array}{c}
V \\
2
\end{array}\right): M \cap M^{\prime} \neq \emptyset\right\}\right\} .
$$

In words, the vertices of each graph in $\operatorname{IG}(\mathcal{M})$ are sets in $\mathcal{M}$, and two vertices are connected by an edge if they have a nonempty intersection.

Usually we consider intersection graphs of $\mathcal{M}$ up to isomorphism; i.e., we regard a graph $G$ as an intersection graph of $\mathcal{M}$ if it is merely isomorphic to a graph $G^{\prime} \in \operatorname{IG}(\mathcal{M})$. In that case we call $V\left(G^{\prime}\right) \subseteq \mathcal{M}$ an $\mathcal{M}$-representation of $G$, or just a representation of $G$ if $\mathcal{M}$ is understood.

\section{Important examples.}

- For $\mathcal{M}$ consisting of all (closed) intervals on the real line, we obtain the class of interval graphs. This is one of the most useful graph classes in applications. Interval graphs have several characterizations, they can be recognized in linear time, and there is even a detective story Who Killed the Duke of Densmore? by Claude Berge (in French; see [Ber95] for English translation) in which the solution depends on properties of interval graphs.

- Disk graphs, i.e., intersection graphs of disks in the plane, and unit disk graphs have been studied extensively. Of course, one can also investigate intersection graphs of balls in $\mathbb{R}^{d}$ for a given $d$, or of unit balls.

- Another interesting class is CONV, the intersection graphs of convex sets in the plane.

- Here we will devote most of the time to the class STRING of string graphs, the intersection graphs of simple curves in the plane.

- Another important class is SEG, the segment graphs, which are the intersection graphs of line segments in $\mathbb{R}^{2}$.

Other interesting classes of graphs are obtained by placing various restrictions on the mutual position or intersection pattern of the sets representing the vertices. For example:

- For an integer $k \geq 1, k$-STRING is the subclass of string graphs consisting of all graphs representable by curves such that every two of them have at most $k$ points of intersection. $]^{1}$

\footnotetext{
${ }^{1}$ Some authors moreover require that each of the intersection points is a crossing, i.e., a point where, locally, one of the edges passes from one side of the second edge to the other (as opposed to a touching point).
} 
- For $k \geq 1$, the class $k$-DIR consists of the segment graphs possessing a representation in which the segments involved have at most $k$ distinct directions. (So 1-DIR are just interval graphs.)

- The kissing graphs of circles, sometimes also called contact graphs of circles or coin graphs, are disk graphs that admit a representation by disks with disjoint interiors; that is, every two disks either are disjoint or just touch. The beautiful and surprisingly useful Koebe-AndreevThurston theorem asserts that a graph is a kissing graph of circles if and only if it is planar. While "only if" is easy to see, the "if" direction is highly nontrivial. Here we have just mentioned this gem of a result but we will not discuss it any further.

Typical questions. For each class $\mathcal{C}$ of intersection graphs, and in particular, for all the classes mentioned above, one can ask a number of basic questions. Here are some examples:

- How hard, computationally, is the recognition problem for $\mathcal{C}$ ? That is, given an (abstract) graph $G$, is it isomorphic to a graph in $\mathcal{C}$ ? For some classes, such as the interval graphs, polynomial-time or even linear-time algorithms have been found, while for many other classes the recognition problem has been shown NP-hard, and sometimes it is suspected to be even harder (not to belong to NP).

- How complicated a representation may be required for the graphs in $\mathcal{C}$ ? In more detail, we first need to define some reasonable notion of size of a representation of a graph in $\mathcal{C}$. Then we ask, given an integer $n$, what is the maximum, over all $n$-vertex graphs $G \in \mathcal{C}$, of the smallest possible size of a representation of $G$ ?

For example, it is not too difficult to show that each segment graph has a representation in which all of the segments have endpoints with integral coordinates. For such a representation, the size can be defined as the total number of bits needed for encoding all the coordinates of the endpoints.

- Can the chromatic number be bounded in terms of the clique number? It is well known that there are graphs $G$ with clique number $\omega(G)=2$, i.e., triangle-free, and with chromatic number $\chi(G)$ arbitrarily large. On the other hand, many important classes, such as interval graphs, consist of perfect graphs, which satisfy $\omega(G)=\chi(G)$. Some classes $\mathcal{C}$ display an intermediate behavior, namely, $\chi(G) \leq f(\omega(G))$ for all $G \in \mathcal{C}$ and for some function $f: \mathbb{N} \rightarrow \mathbb{N}$; establishing a bound of this kind is often of considerable interest, and then one may ask for the smallest possible $f$. If $\chi(G)$ cannot be bounded in terms of $\omega(G)$ alone, one may still investigate bounds for $\chi(G)$ in terms of $\omega(G)$ and the number of vertices of $G$.

- One may also consider two classes of interest, $\mathcal{C}$ and $\mathcal{C}^{\prime}$, and ask for inclusion relations among them (e.g., whether $\mathcal{C} \subseteq \mathcal{C}^{\prime}$, or $\mathcal{C}^{\prime} \subseteq \mathcal{C}$, or even $\left.\mathcal{C}=\mathcal{C}^{\prime}\right)$. Some relations are quite easy, such as SEG $\subseteq$ CONV $\subseteq$ STRING, but others may be very challenging. 
For example, Scheinermann conjectured in his $\mathrm{PhD}$. thesis in 1984 that all planar graphs are in SEG. It took over 20 years until Chalopin, Gonçalves, and Ochem [CGO10] managed to prove the weaker result that all planar graphs are in 1-STRING, and in 2009 Chalopin and Gonçalves [CG09] finally established Scheinermann's conjecture.

Thus, it should already be apparent from our short lists of classes and questions that the study of intersection graphs is an area in which it is very easy to produce problems (and exercises). However, instead of trying to survey the area, we will focus on a small number of selected results. Some of them also serve us as a stage on which we are going to show various interesting tools in action.

Exercise 1.1 Prove carefully an assertion made above: every SEG-graph has a representation with all segment endpoints integral. (Hint: check the definition of SEG again and note what it does not assume.)

Exercise 1.2 Show that graphs in 100-STRING can be recognized in NP.

\section{Basics of string graphs}

We begin with a trivial but important observation: all of the complete graphs $K_{n}$ are string graphs. Hence, unlike classes such as planar graphs, string graphs can be dense and they have no forbidden minors. Moreover, they are not closed under taking minors; thus, the wonderful Robertson-Seymour theory is not applicable.

Another simple observation asserts that every planar graph is a string graph, even 2-STRING. The following picture indicates the proof:
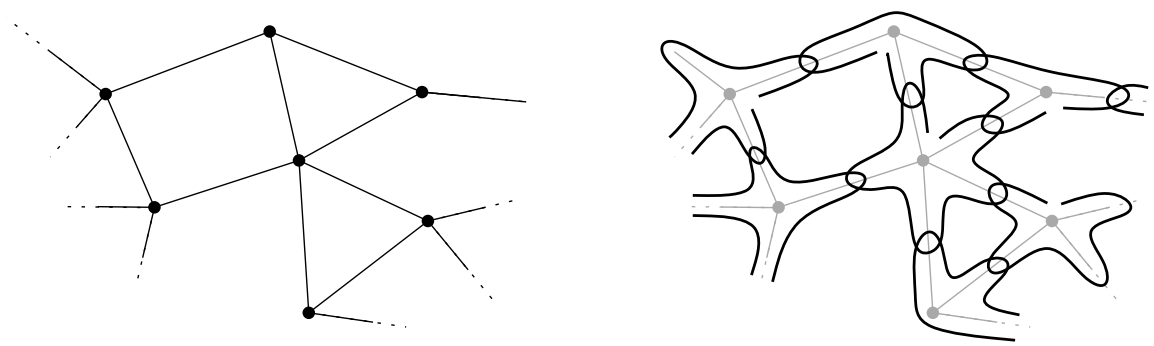

As we have mentioned, every planar graph is even a segment graph, but this is a difficult recent result [CG09.

Example 2.1 It is not completely easy to come up with an example of a nonstring graph. Here is one:

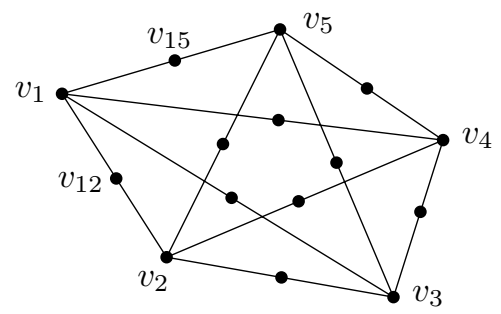


(more generally, every graph obtained from a non-planar graph by replacing each edge by a path of length at least two is non-string).

Sketch of proof. For contradiction we suppose that this graph has a representation by simple curves (referred to as strings in this context), where each $v_{i}$ is represented by a string $\gamma_{i}$ and $v_{i j}$ is represented by $\gamma_{i j}$. From such a representation we will obtain a planar drawing of $K_{5}$, thus reaching a contradiction.

To this end, we first select, for each $i<j$, a piece $\pi_{i j}$ of $\gamma_{i j}$ connecting a point of $\gamma_{i}$ to a point of $\gamma_{j}$ and otherwise disjoint from $\gamma_{i}$ and $\gamma_{j}$. Next, we continuously shrink each $\gamma_{i}$ to a point, pulling the $\pi_{i j}$ along - the result is the promised planar drawing of $K_{5}$. The picture shows this construction in the vicinity of the string $\gamma_{1}$ :
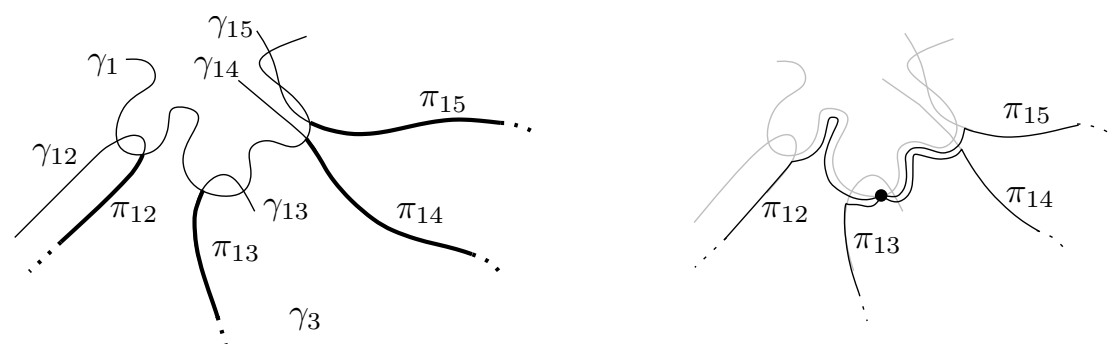

Admittedly, this argument is not very rigorous, and if the strings are arbitrary curves, it is difficult to specify the construction precisely. An easier route towards a rigorous proof hinges on the following generally useful fact.

Lemma 2.2 Every (finite) string graph $G$ can be represented by polygonal curves, i.e., simple curves consisting of finitely many segments. We may also assume that every two curves have finitely many intersection points, and that no point belongs to three or more curves.

Sketch of proof. We start from an arbitrary string representation of $G$. By compactness, there exists an $\varepsilon>0$ such that every two disjoint strings in the representation have distance at least $\varepsilon$. For every two strings $\gamma, \delta$ that intersect, we pick a point $p_{\gamma \delta}$ in the intersection. Then we replace each string $\gamma$ by a polygonal curve that interconnects all the points $p_{\gamma \delta}$ and lies in the open $\frac{\varepsilon}{2}$-neighborhood of $\gamma$.

By a small perturbation of the resulting polygonal curves we can then achieve finitely many intersections and eliminate all triple points.

Exercise 2.3 Let $U \subseteq \mathbb{R}^{2}$ be an open, arcwise connected set; that is, every two points of $U$ can be connected by a simple curve in $U$. Prove, as rigorously as possible, that every two points of $U$ can also be connected by a polygonal curve.

Let us call a string representation as in Lemma 2.2 standard. 


\section{String graphs requiring exponentially many inter- sections}

How hard is to recognize string graphs? Using an ingenious reduction, Kratochvíl [Kra91] proved that recognizing string graphs is NP-hard, but the question remained, does this problem belong to the class NP?

A natural way of showing membership of the problem in NP would be to guess a string representation, and verify in polynomial time that it indeed represents a given graph $G$. A simple way of specifying a string representation is to put a vertex into every intersection point of the strings, and describe the resulting plane (multi)graph:

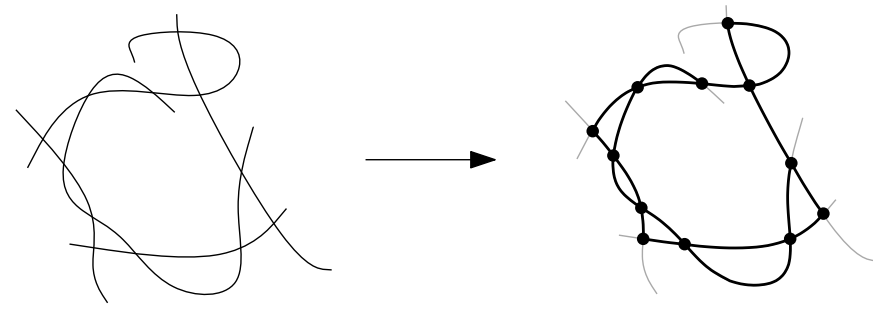

In this description, the edges are labeled by the strings they come from. Then it can be checked whether such a plane graph indeed provides a string representation of $G$.

This argument may seem to prove membership in NP easily, but there is a catch: namely, we would need to know that there is a polynomial $p(n)$ such that every string graph on $n$ vertices admits a string representation with at most $p(n)$ intersection points. However, as was noticed in [KM91], this is false - as we will prove below, there are string graphs for which every representation has exponentially many intersections. After this result, for ten years it was not clear whether there is any algorithm at all for recognizing string graphs.

Weak realizations. As an auxiliary device, we introduce the following notions. An abstract topological graph is a pair $(G, R)$, where $G$ is an (abstract) graph and $R \subseteq\left(\begin{array}{c}E(G) \\ 2\end{array}\right)$ is a symmetric relation on the edge set. A weak realization of such $(G, R)$ is a drawing of $G$ in the plane such that whenever two edges $e, e^{\prime}$ intersect (sharing a vertex does not count), we have $\left\{e, e^{\prime}\right\} \in R$. Thus, $R$ specifies which pairs of edges are allowed (but not forced) to intersect.

We call a weak realization standard if the corresponding drawing of $G$ is standard, by which we mean that the edges are drawn as polygonal curves, every two intersect at finitely many points, and no three edges have a common intersection (where sharing a vertex does not count). (Moreover, as in every graph drawing we assume that the edges do not pass through vertices.) Standard drawings help us to get rid of "local" difficulties in proofs.

Exercise 3.1 (a) Prove that if $(G, R)$ has a weak realization, then it also has a standard weak realization. (This is analogous to Lemma 2.2, but extra care is needed near the vertices!) 
(b) Prove that if $(G, R)$ has a weak realization $W$ with finitely many edge intersections in which no three edges have a common intersection, then it also has a standard weak realization $W^{\prime}$ with at most as many edge intersections as in $W$.

For a string graph $G$, let $f_{s}(G)$ denote the minimum number of intersection points in a standard string representation of $G$, and let

$$
f_{s}(n):=\max \left\{f_{s}(G): G \text { a string graph on } n \text { vertices }\right\} .
$$

Similarly, for an abstract topological graph $(G, R)$ admitting a weak realization, let $f_{w}(G, R)$ be the minimum number of edge intersections in a standard weak realization of $(G, R)$, and

$$
f_{w}(m):=\max \left\{f_{w}(G, R):(G, R) \text { weakly realizable, }|E(G)|=m\right\} .
$$

Observation $3.2 f_{w}(m) \leq f_{s}(2 m)$.

Proof. Let $(G, R)$ be an abstract topological graph with $m$ edges witnessing $f_{w}(m)$. We may assume that $G$ is connected and non-planar (why?), and thus $m \geq n=|V(G)|$.

We consider a (standard) weak realization $W$ of $(G, R)$ with $f_{w}(m)$ intersections, and construct a string representation of a string graph $H$ as follows: we replace every vertex in $W$ by a tiny vertex string, and every edge by an edge string, as is indicated below:

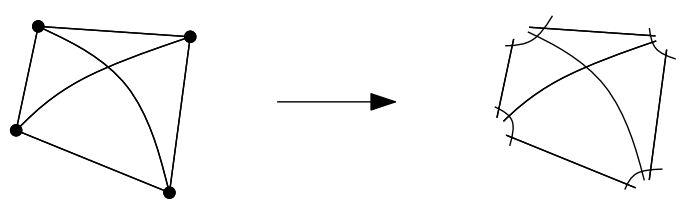

This $H$ has $m+n \leq 2 m$ vertices, and using the monotonicity of $f_{s}$, it suffices to show that $f_{s}(H) \geq f_{w}(m)$. This follows since a string representation of $H$ with $x$ intersections yields a weak realization of $(G, R)$ with at most $x$ intersections, by contracting the vertex strings to points and pulling the edge strings along (this is the same argument as in Example 2.1.

Exercise 3.3 Prove that $f_{s}(n) \leq f_{w}\left(n^{2}\right)+n^{2}$. $\left(\right.$ Or $f_{s}(n) \leq f_{w}\left(O\left(n^{2}\right)\right)+O\left(n^{2}\right)$ if this looks easier.)

Theorem 3.4 There is a constant $c>0$ such that $f_{w}(m) \geq 2^{c m}$, and consequently, $f_{s}(n) \geq 2^{(c / 2) n}$.

Proof. For $k \geq 1$, we define a planar graph $P_{k}$ according to the following picture: 


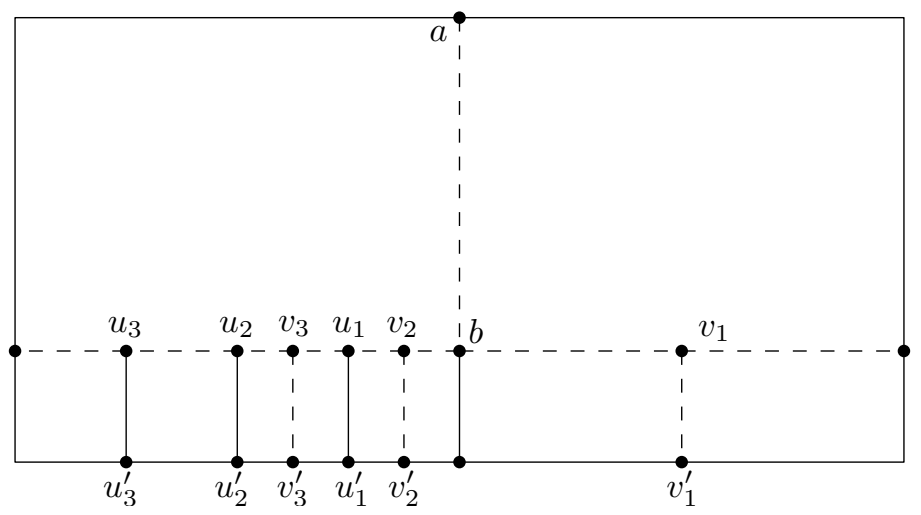

( $P_{k}$ is obtained from $P_{k-1}$ by adding vertices $u_{k}$ and $v_{k}$ to the left and right of $u_{k-1}$, respectively, and adding the vertical edges $\left\{u_{k}, u_{k}^{\prime}\right\}$ and $\left.\left\{v_{k}, v_{k}^{\prime}\right\}\right)$. Then we create an abstract topological graph $\left(G_{k}, R_{k}\right)$ from $P_{k}: G_{k}$ is obtained from $P_{k}$ by adding the edges $\left\{u_{1}, v_{1}\right\}, \ldots,\left\{u_{k}, v_{k}\right\}$, and the relation $R_{k}$ allows each of the edges $\left\{u_{i}, v_{i}\right\}$ to intersect all of the edges drawn dashed in the picture above. No other edge intersections are permitted.

Each $\left(G_{k}, R_{k}\right)$ has a weak realization:

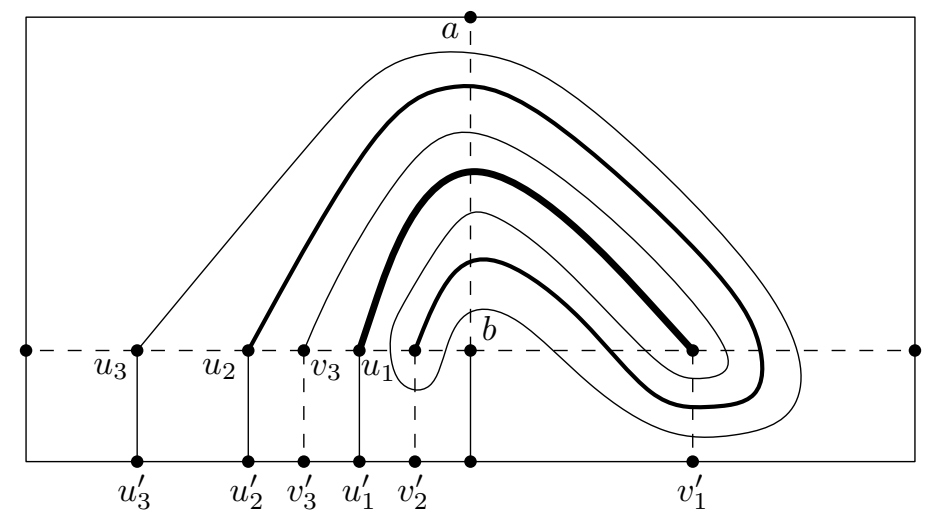

We prove by induction on $i$ that in every weak realization of $G_{k}$, the edge $\left\{u_{i}, v_{i}\right\}$ intersects $\{a, b\}$ at least $2^{i-1}$ times, $1 \leq i \leq k$; then the theorem will follow.

Since $P_{k}$ is a 3 -connected graph, it has a topologically unique drawing. From this the case $i=1$ can be considered obvious. For $i \geq 2$, the situation for the edge $\left\{u_{i}, v_{i}\right\}$ looks, after contracting the edge $\left\{u_{i-1}, u_{i-1}^{\prime}\right\}$ and a simplification preserving the topology, as follows:

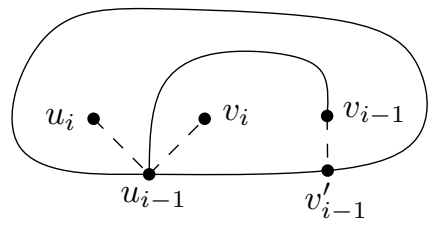

Thus, the edge $\left\{u_{i}, v_{i}\right\}$ has to cross $\left\{v_{i-1}, v_{i-1}^{\prime}\right\}$.

Now we will use the drawing of $\left\{u_{i}, v_{i}\right\}$ to get two different curves $\pi_{1}, \pi_{2}$ that both "duplicate" the previous edge $\left\{u_{i-1}, v_{i-1}\right\}$. The first curve $\pi_{1}$ starts 
at $u_{i-1}$ and follows $\left\{u_{i-1}, u_{i}\right\}$ up to the point where $\left\{u_{i}, v_{i}\right\}$ intersects $\left\{u_{i-1}, u_{i}\right\}$ the last time before hitting $\left\{v_{i-1}, v_{i-1}^{\prime}\right\}$ (that point can also be $u_{i}$ ). Then $\pi_{1}$ follows $\left\{u_{i}, v_{i}\right\}$ almost up to the first intersection with $\left\{v_{i-1}, v_{i-1}^{\prime}\right\}$, and finally, it goes very near $\left\{v_{i-1}, v_{i-1}^{\prime}\right\}$ until $v_{i-1}$ :

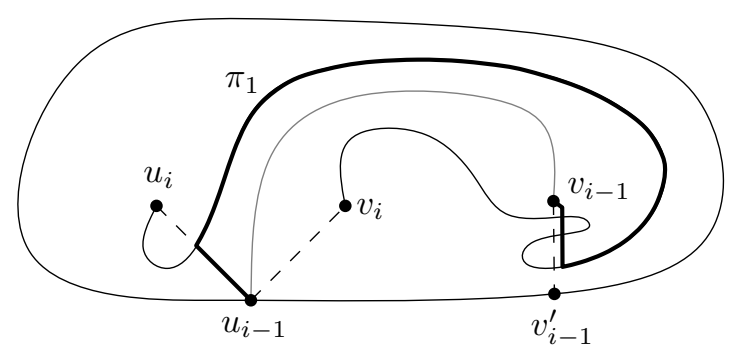

If we remove the drawings of the edges $\left\{u_{j}, v_{j}\right\}, j \geq i-1$, from the considered weak realization of $G_{k}$, and add $\pi_{1}$ as a new way of drawing the edge $\left\{u_{i-1}, v_{i-1}\right\}$, we obtain a weak realization of $G_{i-1}$. Therefore, by the inductive hypothesis, $\pi_{1}$ crosses $\{a, b\}$ at least $2^{i-2}$ times.

Similarly we construct $\pi_{2}$, disjoint from $\pi_{1}$, which starts at $u_{i-1}$ and first follows $\left\{u_{i-1}, v_{i}\right\}$. It again has to cross $\{a, b\}$ at least $2^{i-2}$ times, and the induction step is finished.

\section{Exponentially many intersections suffice}

The first algorithm for recognizing string graphs was provided by Schaefer and Štefankovič [SŠ04], who proved an upper bound on the number of intersections sufficient for a representation of every $n$-vertex string graph. Similar to the previous section, their proof works with weak representations.

Theorem 4.1 ([SŠ04]) We have $f_{w}(m) \leq m 2^{m}$. Consequently (by Exercise 3.3), $f_{s}(n)=2^{O\left(n^{2}\right)}$.

This result implies, by the argument given at the beginning of Section 3 , that string graphs can be recognized in NEXP (nondeterministic exponential time).

Later Schaefer, Sedgwick, and Štefankovič [SSŠ03] proved that string graphs can even be recognized in NP. The main idea of their ingenious argument is that, even though a string representation may require exponentially many intersections, there is always a representation admitting a compact encoding, of only polynomial size, by something like a context-free grammar. They also need to show that, given such a compact encoding of a collection of strings, one can verify in polynomial time whether it represents a given graph. We will not discuss their proof any further and we proceed with a proof of Theorem 4.1.

Let $(G, R)$ be a weakly realizable abstract topological graph with $m$ edges. It has a standard weak realization (edges are polygonal curves with finitely many intersections, and no triple intersections; see Exercise 3.1). Moreover, we can make sure that the edges cross at every intersection point, since a "touching 
point" $\searrow$ can be perturbed away: $\_$(note the advantage of working with weak realizations, in which we need not worry about losing intersections).

Theorem 4.1 is an immediate consequence of the following claim: if $W$ is a standard weak realization in which some edge e has at least $2^{m}$ crossings, then there is another standard weak realization $W^{\prime}$ with fewer crossing than in $W$.

Lemma 4.2 If an edge $e$ has at least $2^{m}$ crossings, then there is a contiguous segment $\hat{e}$ of e that contains at least one crossing and that crosses every edge of $G$ an even number of times.

Proof. The lemma is an immediate consequence of the following combinatorial statement: If $w$ is a word (finite sequence) of length $2^{m}$ over an m-letter alphabet $\Sigma$, then there is a nonempty subword (contiguous subsequence) $x$ of $w$ in which each symbol of $\Sigma$ occurs an even number of times.

To prove this statement, let us define, for $i=0,1, \ldots, 2^{m}$, a mapping $f_{i}: \Sigma \rightarrow\{0,1\}$, where $f_{i}(a)=0$ if $a$ occurs an even number of times among the first $i$ symbols of $w$, and $f_{i}(a)=1$ otherwise. Since there are only $2^{m}$ distinct mappings $\Sigma \rightarrow\{0,1\}$, there are two indices $i \neq j$ with $f_{i}=f_{j}$. Then the subword of $w$ beginning at position $i+1$ and ending at position $j$ is the desired $x$.

Now we fix $e$ and $\hat{e}$ as in the lemma. We can deform the plane by a suitable homeomorphism so that $\hat{e}$ is a horizontal straight segment and there is a narrow band along it, which we call the window, in which the edges crossing $\hat{e}$ appear as little vertical segments, and in which no other portions of the edges are present:

\begin{tabular}{c|cccccccccccc}
$f$ & $g$ & $f$ & $h$ & $g$ & $g$ & $f$ & $f$ & $g$ & $f$ & $h$ & $f$ \\
\hline$\hat{e}$ & & & $\vdots$ & & 1 & & & 1 & $\vdots$ & \\
\hline & & & $\vdots$ & 1 & 1 & & & 1 & & $\vdots$ & \\
\hline
\end{tabular}

Any edge $f \neq e$ has an even number $2 n_{f}$ crossings with $\hat{e}$, and it intersects the border of the window in $4 n_{f}$ points. Let us number these $4 n_{f}$ points as $p_{f, 1}, \ldots, p_{f, 4 n_{f}}$ in the order as they appear along $f$ (we choose one of the two possible directions of traversing $f$ arbitrarily).

Here is the procedure for redrawing the original weak representation $W$ into $W^{\prime}$ so that the total number of crossings is reduced.

1. We apply a suitable homeomorphism of the plane that maps the window to a circular disk with $\hat{e}$ as the horizontal diameter, while the edges crossing $\hat{e}$ still appear as vertical segments within the window. For every $f$ and every $i=1,2, \ldots, 2 n_{f}-1$, the point $p_{f, 2 i}$ is connected to $p_{f, 2 i+1}$ by an arc of $f$ outside the window. Let us call these arcs for $i$ odd the odd connectors and for $i$ even the even connectors. The following illustration shows only one edge $f$, although the window may be intersected by many edges. The points $p_{f, i}$ are labeled only by their indices, and the odd connectors are drawn thick: 


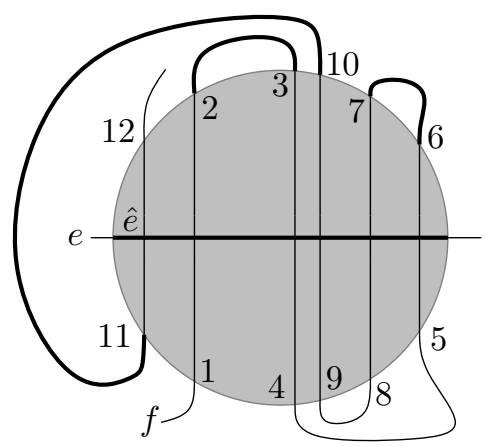

2. We erase everything inside the window. Then we map the odd connectors inside the window by the circular inversion that maps the outside of the window to its inside, while the even connectors stay outside. Crucially, two odd connectors that did not intersect before the circular inversion still do not intersect. Next, we apply the mirror reflection about $\hat{e}$ inside the window to the odd connectors:
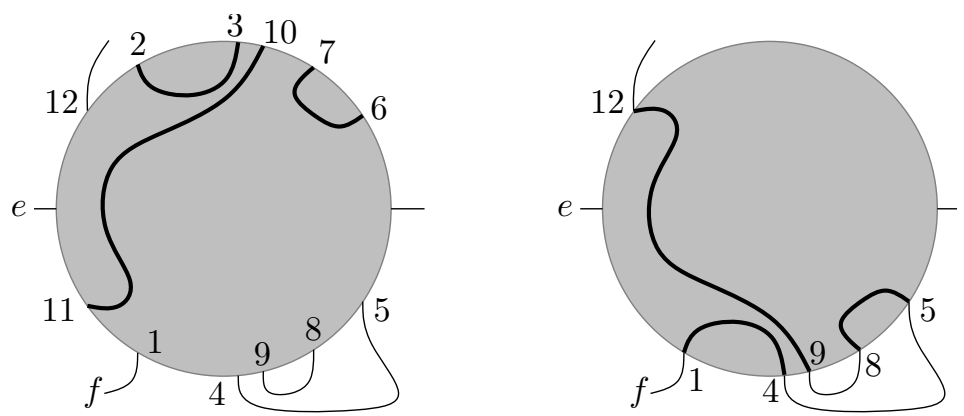

As the picture illustrates, these transformed odd connectors together with the original even connectors connect up the initial piece of $f$ to the final piece. This new way of drawing of $f$ crosses the window $n_{f}$ times, only half of the original number. This is the moment where we use the fact that each edge crosses $\hat{e}$ an even number of times; otherwise, the re-connection of $f$ would not work.

After this redrawing of all the edges crossing $\hat{e}$, edges that did not cross before still do not cross (while some intersections may be lost).

3. It remains to draw the erased portion of $e$. We do not want to draw it horizontally, since we would have no control over the intersections with the transformed odd connectors. Instead, we draw it along the top or bottom half-circle bounding the window, whichever gives a smaller number of intersections (breaking a tie arbitrarily). 


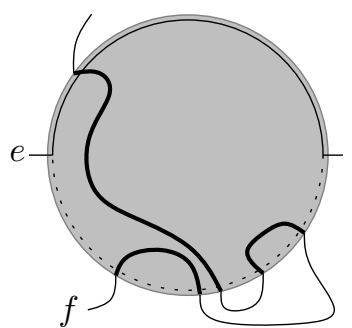

Each $f$ crosses the window border $2 n_{f}$ times after the redrawing, and thus one of the half-circles is crossed at most $\sum_{f} n_{f}$ times - while originally $\hat{e}$ was crossed $2 \sum_{f} n_{f}$ times. Hence the redrawing indeed reduces the number of crossings. The resulting weak realization is not necessarily standard, but we can make it standard without increasing the number of intersections (Exercise 3.1(b)), and Theorem 4.1 is proved.

\section{A separator theorem for string graphs}

Let $G$ be a graph. A subset $S \subseteq V(G)$ is called a separator if there is a partition of $V(G) \backslash S$ into disjoint subsets $A$ and $B$ such that there are no edges between $A$ and $B$ and $|A|,|B| \leq \frac{2}{3}|V(G)|$.

Exercise 5.1 Check that the above definition of a separator is equivalent to requiring all connected components of $G \backslash S$ to have at most $\frac{2}{3}|V(G)|$ vertices.

Exercise 5.2 (a) Show that every tree has a one-vertex separator.

(b) We could also define a $\beta$-separator, for $\beta \in(0,1)$, by replacing $\frac{2}{3}$ in the above definition by $\beta$. Check that for $\beta<\frac{2}{3}$, there are trees with no onevertex $\beta$-separator. From this point of view, the value $\frac{2}{3}$ is natural. (For most applications, though, having $\beta$-separators for a constant $\beta<1$ is sufficient, and the specific value of $\beta$ is not too important.)

Separator theorems are results asserting that all graphs in a certain class have "small" separators (much smaller than the number of vertices). They have lots of applications, and in particular, they are the basis of many efficient divide-and-conquer algorithms.

Probably the most famous separator theorem, and arguably one of the nicest and most useful ones, is the Lipton-Tarjan separator theorem for planar graphs, asserting that every planar graph on $n$ vertices has a separator of size $O(\sqrt{n})$.

Exercise 5.3 Show that the $m \times m$ square grid has no separator of size $m / 4$. Thus, the order of magnitude in the Lipton-Tarjan theorem cannot be improved.

The separator theorem has several proofs (let us mention a simple graphtheoretic proof by Alon et al. [AST94] and a neat proof from the KoebeAndreev-Thurston theorem mentioned in Section 1; see, e.g., [PA95]). There are scores of generalizations and variations. For example, every class of graphs with a fixed excluded minor admits $O(\sqrt{n})$-size separators AST94. 
Here we focus on a separator theorem for string graphs. Of course, in this case we cannot bound the separator size by a sublinear function of the number of vertices (because $K_{n}$ is a string graph); for this reason, the bound is in terms of the number of edges.

Theorem 5.4 Every string graph with $m \geq 2$ edges has a separator with $O(\sqrt{m} \log m)$ vertices.

The first separator theorem for string graphs, with a worse bound of $O\left(m^{3 / 4} \sqrt{\log m}\right)$, was proved by Fox and Pach [FP10]. The improvement to $O(\sqrt{m} \log m)$ was obtained while preparing this text, and it was published in a concise form in Mat14.

The proof, whose exposition will occupy most of the rest of this chapter, is a remarkable chain of diverse ideas coming from various sources.

Fox and Pach conjectured that the theorem should hold with $O(\sqrt{m})$. This, if true, would be asymptotically optimal: we already know this for graphs with $n$ vertices and $O(n)$ edges, since every planar graph is a string graph, but asymptotic optimality holds for graphs with any number of edges between $n$ and $\left(\begin{array}{l}n \\ 2\end{array}\right)$.

The separator theorem shows that string graphs are (globally) very different from typical (not too dense) random graphs and, more generally, from expanders.

In the next section, we demonstrate a surprising use of Theorem 5.4 for a number of other applications we refer to [FP10, FP14]. Then we start working on the proof of Theorem 5.4 in Section 7.

\section{Crossing number versus pair-crossing number}

Now something else: we will discuss crossing numbers in this section. The crossing number $\operatorname{cr}(G)$ of a graph $G$ is the smallest possible number of edge intersections (crossings) in a standard drawing of $G$. We recall that in a standard drawing, edges are polygonal lines with finitely many intersections and no triple points; see Section 3. In this section we consider only standard drawings.

One may also consider the rectilinear crossing number $\overline{\mathrm{cr}}(G)$, which is the minimum number of crossings in a straight-edge drawing of $G$, but this behaves very differently from $\operatorname{cr}(G)$, and the methods involved in its study are also different from those employed for the crossing number.

An algorithmic remark. The crossing number is an extensively studied and difficult graph parameter. Let us just mention in passing that computing $\operatorname{cr}(G)$ is known to be NP-complete, but a tantalizing open problem is, how well it can be approximated (in polynomial time). On the one hand, there is a constant $c>1$ such that $\operatorname{cr}(G)$ is hard to approximate within a factor of $c$ [Cab13, and on the other hand, there is a (highly complicated) algorithm Chu11 with approximation factor roughly $n^{9 / 10}$ for $n$-vertex graphs with maximum degree bounded by a constant. The latter result may not look very impressive, but it is the first one breaking a long-standing barrier of $n$. 
A difficult case here are graphs with relatively small, but not too small, crossing number, say around $n$. Indeed, on the one hand, for every fixed $k$, there is a linear-time algorithm deciding whether $\operatorname{cr}(G) \leq k$ KR07, Gro04. On the other hand, for a graph with maximum degree bounded by a constant and with crossing number $k$, a drawing with at most $O\left((n+k)(\log n)^{2}\right)$ crossings can be found in polynomial time; this is based on [LR99], with improvements of [EGS03, ARV09] (also see [CMS11]).

The single-crossing lemma. Here is a useful basic fact about drawings minimizing the crossing number.

Lemma 6.1 (Single-crossing lemma) In every (standard) drawing of $G$ that minimizes the crossing number, no two edges intersect more than once.

Proof. We show that if edges $e, e^{\prime}$ intersect at least twice, the number of crossings can be reduced. We consider crossings $X_{1}$ and $X_{2}$ that are consecutive along $e$. There are two cases to consider, the second one being easy to overlook, and in each of them we redraw the edges locally as indicated:
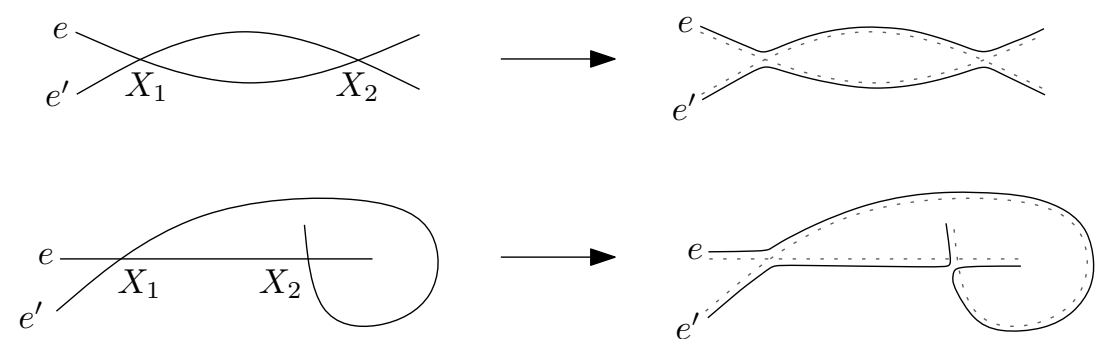

This reduces the total number of crossings by at least 2 . We also note that since the part of $e^{\prime}$ between $X_{1}$ and $X_{2}$ may be intersected by $e$, this redrawing may introduce self-intersections of $e$ - but these are easily eliminated by shortcutting the resulting loops on $e$.

Exercise 6.2 Let us say that $X_{1}, X_{2}$ is a simple pair of crossings of edges e and $e^{\prime}$ if $X_{1}$ and $X_{2}$ are consecutive on both $e$ and $e^{\prime}$. Draw an example of two edges that cross several times but that have no simple pair of crossings.

The lemma just proved shows that it does not matter whether we define the crossing number as the minimum number of crossings or as the minimum number of crossing pairs of edges in a drawing. Do you believe the previous sentence? If yes, you are in a good company, since many people got caught, and only Pach and Tóth [PT00, and independently Mohar (at a 1995 AMS Conference on Topological Graph Theory), noticed that the lemma proves nothing like that, since it is not clear whether a drawing with minimum number of crossings also has a minimum number of crossing pairs. Indeed, the number of crossing pairs may very well increase in a redrawing as in the proof of the lemma:

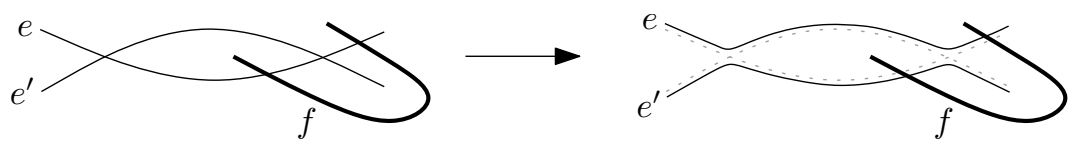


Thus, it makes good sense to define the pair-crossing number $\operatorname{pcr}(G)$ as the minimum possible number of pairs of edges that cross in a drawing of $G$. Clearly, $\operatorname{pcr}(G) \leq \operatorname{cr}(G)$ for all $G$.

It is generally conjectured that $\operatorname{pcr}(G)=\operatorname{cr}(G)$ for all $G$, but if true, this is unlikely to be proved by a "local" redrawing argument in the spirit of Lemma 6.1 at least, many people tried that and failed.

A warning example is a result of Pelsmajer et al. [PSŠ08], who found a graph $G$ with $\operatorname{ocr}(G)<\operatorname{cr}(G)$. Here $\operatorname{ocr}(G)$ is the odd crossing number of $G$, which is the minimum number, over drawings of $G$, of pairs of edges that cross an odd number of times. A good motivation for studying the odd crossing number is the famous Hanani-Tutte theorem, asserting that if a graph has a drawing in which every two non-adjacent edges cross an even number of times, then it is planar (see [Sch14] for a modern treatment). In particular, this implies that $\operatorname{ocr}(G)=0, \operatorname{pcr}(G)=0$, and $\operatorname{cr}(G)=0$ are all equivalent.

One direction of investigating the pcr/cr puzzle is to bound the crossing number by some function of the pair-crossing number, and to try to get as small a bound as possible. We begin with a simple result in this direction.

Proposition 6.3 If $\operatorname{pcr}(G)=k$, then $\operatorname{cr}(G) \leq 2 k^{2}$.

Proof. We fix a drawing $D$ of $G=(V, E)$ witnessing $\operatorname{pcr}(G)$. Let $F \subseteq E$ be the set of edges that participate in at least one crossing, and let $E_{0}=E \backslash F$; thus, the edges of $E_{0}$ define a plane subgraph in $D$. We keep the drawing of these edges and we redraw the edges of $F$ so that every two of them have at most one crossing, as in the proof of Lemma 6.1 (we note that $E_{0}$ does not interfere with this redrawing in any way). Since $\mid \overrightarrow{F \mid} \leq 2 k$, the resulting drawing has at most $\left(\begin{array}{c}2 k \\ 2\end{array}\right) \leq 2 k^{2}$ crossings.

The current strongest bound is based on the separator theorem for string graphs.

Theorem 6.4 If $\operatorname{pcr}(G)=k \geq 2$, then $\operatorname{cr}(G)=O\left(k^{3 / 2}(\log k)^{2}\right)$.

The following proof is due to Tóth [Tót12]; he states a worse bound, but the bound above follows immediately from his proof by plugging in a better separator theorem.

We begin the proof with a variant of the single-crossing lemma (Lemma 6.1).

Lemma 6.5 (Red-blue single-crossing lemma) Let $G$ be a graph in which each edge is either red or blue, and let $D$ be a drawing of $G$. Then there is a drawing $D^{\prime}$ of $G$ such that the following hold:

(i) Each edge in $D^{\prime}$ is drawn in an arbitrarily small neighborhood of the edges of $D$.

(ii) Every two edges intersect at most once in $D^{\prime}$.

(iii) The number of blue-blue crossings in $D^{\prime}$ is no larger than in $D$. 
Proof. While edges $e, e^{\prime}$ crossing at least twice exist, we repeat redrawing operations similar to those in Lemma 6.1. However, while in that lemma we swapped portions of $e$ and $e^{\prime}$, here we keep $e$ fixed and route $e^{\prime}$ along it,
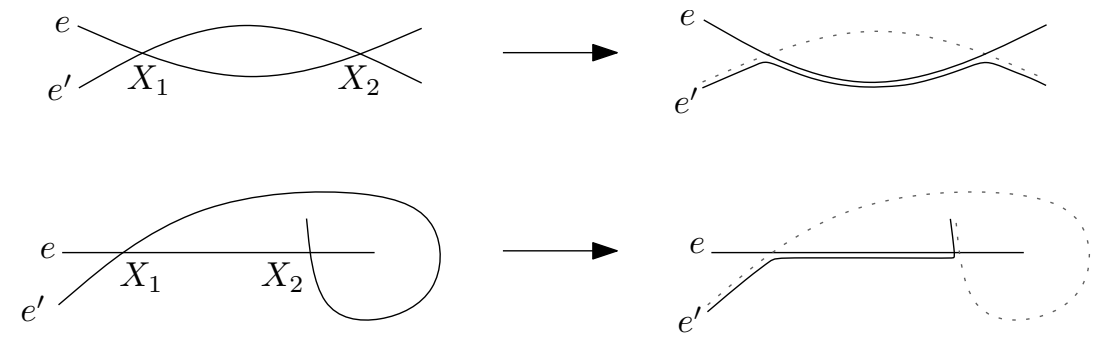

or the other way round. We may again introduce self-intersections of $e$ or $e^{\prime}$, but we remove them by shortcutting loops.

To decide which way of redrawing to use, we let $\hat{e}$ be the portion of $e$ between $X_{1}$ and $X_{2}$ (excluding $X_{1}$ and $X_{2}$ ), and similarly for $\hat{e}^{\prime}$. Let $b$ and $b^{\prime}$ be the number of crossings of blue edges with $\hat{e}$ and $\hat{e}^{\prime}$, respectively, and similarly for $r, r^{\prime}$. If the pair $(b, r)$ is lexicographically smaller than $\left(b^{\prime}, r^{\prime}\right)$, we route $\hat{e}^{\prime}$ along $\hat{e}$, and otherwise, $\hat{e}$ along $\hat{e}^{\prime}$.

It is clear that if this redrawing procedure terminates, then it yields a drawing $D^{\prime}$ satisfying (i) and (ii). To show that it terminates, and that (iii) holds, it suffices to check that each redrawing strictly lexicographically decreases the vector $\left(x_{B B}, x_{R B}, x_{R R}\right)$ for the current drawing, where $x_{B B}$ is the total number of blue-blue crossings, and similarly for $x_{R B}$ and $x_{R R}$.

To check this, we distinguish three cases. If both $e, e^{\prime}$ are blue, then the redrawing decreases $x_{B B}$. If both $e, e^{\prime}$ are red, then $x_{B B}$ stays the same and either $x_{R B}$ decreases, or it stays the same and $x_{R R}$ decreases. Finally, if $e$ is blue and $e^{\prime}$ is red, then either $x_{B B}$ decreases, or it stays the same and $x_{R B}$ decreases.

In order to prove Theorem 6.4 we will proceed by induction. The inductive hypothesis is the following strengthening of the theorem.

Claim 6.6 Let $G=(V, E)$ be a graph, and let $D$ be a drawing of $G$ with $k$ crossing pairs of edges and with $\ell \geq 2$ edges that have at least one crossing (thus, $\ell \leq 2 k$ and $k \leq\left(\begin{array}{l}\ell \\ 2\end{array}\right)$ ). Then there is a drawing $D^{\prime}$ of $G$ such that every edge is drawn in an arbitrarily small neighborhood of the edges in $D$, and $D^{\prime}$ has at most $A k^{3 / 2}(\log \ell)^{2}$ crossings, where $A$ is a suitable constant.

Proof. We proceed by induction on $\ell$ (the proof will also establish the base case $\ell=2$ directly).

As in the proof of Proposition 6.3, we first partition the edge set $E$ into $F$, the edges with crossings, and $E_{0}=E \backslash F$. Thus, $|F|=\ell$.

Let us consider the edges of $F$ in the drawing $D$ as strings (where we cut off tiny pieces near the vertices, so that the strings meet only if the corresponding edges cross). This defines a string graph with $\ell$ vertices and $k$ edges.

By Theorem 5.4, this string graph has a separator of size at most $C \sqrt{k} \log k$, with a suitable constant $C$. This defines a partition of $F$ into disjoint subsets 
$F_{0}, F_{1}, F_{1}$; we have $\ell_{0}:=\left|F_{0}\right| \leq C \sqrt{k} \log k,\left|F_{1}\right|,\left|F_{2}\right| \leq \frac{2}{3} \ell$, and no edge of $F_{1}$ crosses any edge of $F_{2}$. Let $k_{i}$ be the number of crossing pairs of edges of $F_{i}$ in $D, i=1,2$; we have $k_{1}+k_{2} \leq k$. Let $\ell_{i}$ be the number of edges of $F_{i}$ that cross some edge of $F_{i}$.

Actually, Theorem 5.4 can be applied only if $k \geq 2$, while in our case, for $\ell=2$ it may happen that $k=1$. But if $k=1$, we simply put $F_{0}=F$ and $F_{1}=F_{2}=\emptyset$, and proceed with the subsequent argument.

Next, we apply the inductive hypothesis to the graphs $G_{1}:=\left(V, F_{1}\right)$ and $G_{2}:=\left(V, F_{2}\right)$ (drawn as in $\left.D\right)$. This yields drawings $D_{1}^{\prime}$ and $D_{2}^{\prime}$ as in the claim. (If $F_{1}$ has no crossings then, strictly speaking, the inductive hypothesis cannot be applied, but then $D_{1}^{\prime}$ can be taken as the plane drawing of $G_{1}$ inherited from $D$, and similarly for $F_{2}$.)

For $\ell_{i} \geq 2$, we can bound the number of crossings in $D_{i}^{\prime}$ by $A k_{i}^{3 / 2}\left(\log \ell_{i}\right)^{2}$ according to the inductive hypothesis; for $\ell_{i}=0$ there are no crossings (and $\ell_{i}=1$ is impossible). We have $\ell_{i} \leq\left|F_{i}\right| \leq \frac{2}{3} \ell$, and hence $\log \ell_{i} \leq \log \ell-c_{0}$, where $c_{0}=\log \frac{3}{2}>0$. For the subsequent computation, it will be convenient to bound $\left(\log \ell_{i}\right)^{2}$ from above in a slightly strange-looking way: by $(\log \ell)\left(\log \ell-c_{0}\right)$. The resulting bound $A k_{i}^{3 / 2}(\log \ell)\left(\log \ell-c_{0}\right)$ gives 0 for $k_{i}=0$ and so it is also valid in the case $k_{i}=\ell_{i}=0$.

We overlay $D_{1}^{\prime}$ and $D_{2}^{\prime}$ and add the edges of $F_{0}$ drawn as in $D$; this gives a drawing $\tilde{D}$ of the graph $(V, F)$. Let us color the edges of $F_{1} \cup F_{2}$ blue and those of $F_{0}$ red. By the above, and using $k_{1}^{3 / 2}+k_{2}^{3 / 2} \leq k^{3 / 2}$, the total number of blue-blue crossings in $\tilde{D}$ can be bounded by

$$
A\left(k_{1}^{3 / 2}+k_{2}^{3 / 2}\right)(\log \ell)\left(\log \ell-c_{0}\right) \leq A k^{3 / 2}(\log \ell)^{2}-A c_{0} k^{3 / 2} \log \ell .
$$

The first term of the last expression is the desired bound for the number of all crossings, and thus the second term is the "breathing room" - we need to bound the number of red-blue and red-red crossings by $A c_{0} k^{3 / 2} \log \ell$.

We cannot control the number of red-blue and red-red crossings in $\tilde{D}$, but we apply Lemma 6.5 to the graph $(V, F)$ with the drawing $\tilde{D}$. This provides a new drawing of $(V, F)$, to which we add the edges of $E_{0}$ from the original drawing $D$, and this yields the final drawing $D^{\prime}$. The number of blue-blue crossings in $D^{\prime}$ is no larger than in $\tilde{D}$, and the number of red-blue and red-red crossings is at most $\left|F_{0}\right| \cdot|F|=\ell_{0} \ell$. Using $\ell \leq 2 k$ and $k \leq\left(\begin{array}{l}\ell \\ 2\end{array}\right) \leq \ell^{2}$, we further bound this by $(C \sqrt{k} \log k) 2 k \leq 4 C k^{3 / 2} \log \ell \leq A c_{0} k^{3 / 2} \log \ell$, provided that $A$ was chosen sufficiently large.

This concludes the inductive proof of the claim and thus yields Theorem 6.4.

\section{Multicommodity flows, congestion, and cuts}

We start working towards a proof of the separator theorem for string graphs (Theorem 5.4). The overall scheme of the proof is given at the end of this section, but most of the actual work remains for later sections. 
$\boldsymbol{s}$ - $\boldsymbol{t}$ flows. As a motivation for the subsequent developments, we briefly recall the duality between flows and cuts in graphs. If $G=(V, E)$ is a graph with two distinguished vertices $s$ and $t$, in which every edge has unit capacity, then the maximum amount of flow from $s$ to $t$ equals the minimum number of edges we have to remove in order to destroy all $s$ - $t$ paths. There is also a more general weighted version, in which the capacity of each edge $e$ is a given real number $w_{e} \geq 0$.

Multicommodity flows. Instead of flows between just two vertices, we will use multicommodity flows; namely, we want a unit flow between every pair $\{u, v\}$ of vertices of the considered graph.

Let us remark that instead of requiring unit flow for every pair, we can consider an arbitrary demand function $D:\left(\begin{array}{c}V \\ 2\end{array}\right) \rightarrow[0, \infty)$, specifying some demand $D(u, v)$ on the flow between $u$ and $v$ for every pair $\{u, v\}$. All of the considerations below can be done in this more general setting; we can also put weights on edges and vertices. For simplicity, we stick to the unweighted case, which is sufficient for us; conceptually, the weighted case mostly brings nothing new.

For our purposes, it is convenient to formalize a multicommodity flow as an assignment of nonnegative numbers to paths in the considered graph. Thus, we define $\mathcal{P}$ to be the set of all paths (of nonzero length) in $G$, and a multicommodity flow is a mapping $\varphi: \mathcal{P} \rightarrow[0, \infty)$. Since we will talk almost exclusively about multicommodity flows, we will sometimes say just "flow" instead of "multicommodity flow".

The amount of flow between two vertices $u, v$ is $\sum_{P \in \mathcal{P}_{u v}} \varphi(P)$, where $\mathcal{P}_{u v} \subseteq$ $\mathcal{P}$ is the set of all paths with end-vertices $u$ and $v$. If we think of the vertices as cities and the edges as roads, then $\varphi(P), P \in \mathcal{P}_{u v}$ may be the number of cars per hour driving from $u$ to $v$ or from $v$ to $u$ along the route $P$.

Edge congestion. The requirement of unit flow between every pair of vertices is expressed as $\sum_{P \in \mathcal{P}_{u v}} \varphi(P) \geq 1,\{u, v\} \in\left(\begin{array}{c}V \\ 2\end{array}\right)$. We define the edge congestion under $\varphi$ as

$$
\operatorname{econg}(\varphi)=\max _{e \in E} \sum_{P \in \mathcal{P}: e \in P} \varphi(P),
$$

and the edge congestion of $G$ as $\operatorname{econg}(G)=\min _{\varphi} \operatorname{econg}(\varphi)$, where the minimum is over all flows with unit flow between every two vertices ${ }^{2}$ If $G$ is disconnected, then there are no flows $\varphi$ as above, and we have $\operatorname{econg}(G)=\infty$.

Let us consider an edge cut in $G$, which for us is a partition $(A, V \backslash A)$ of $V$ into two nonempty subsets. By $E(A, V \backslash A)$ we denote the set of all edges of $G$ connecting $A$ to $V \backslash A$. If there is a unit flow between every two vertices of $G$, then $|A| \cdot|V \backslash A|$ units of flow have to pass through the edges of $E(A, V \backslash A)$, and hence

$$
\operatorname{econg}(G) \geq \frac{|A| \cdot|V \backslash A|}{|E(A, V \backslash A)|}
$$

If we define the sparsity of the edge cut $(A, V \backslash A)$ as

$$
\operatorname{espars}(A, V \backslash A)=\frac{|E(A, V \backslash A)|}{|A| \cdot|V \backslash A|},
$$

\footnotetext{
${ }^{2} \mathrm{~A}$ compactness argument, which we omit, shows that the minimum is actually attained.
} 
and the edge sparsity ${ }^{3} \operatorname{espars}(G):=\min _{A} \operatorname{espars}(A, V \backslash A)$, we can write the conclusion of the previous consideration compactly as espars $(G) \geq 1 / \operatorname{econg}(G)$.

Approximate duality. Unlike in the case of $s$ - $t$ flows, it turns out that the last inequality can be strict.

Exercise 7.1 Let $G$ be an n-vertex constant-degree expander, which means that, for some constants $\Delta$ and $\beta>0$, all degrees in $G$ are at most $\Delta$ and $\operatorname{espars}(G) \geq \frac{\beta}{n}$. The existence of such graphs, with some $\Delta, \beta$ fixed and $n$ arbitrarily large, is well known; see, e.g., [HLW06]. Prove that $\operatorname{econg}(G)>$ $1 / \operatorname{espars}(G)$ (assuming that $n$ is sufficiently large in terms of $\Delta$ and $\beta$ ), and actually, that $\operatorname{econg}(G)=\Omega\left(\frac{\log n}{\operatorname{espars}(G)}\right)$. Hint: show that, say, half of the vertex pairs have distance $\Omega(\log n)$.

However, an important result, discovered by Leighton and Rao [LR99], asserts that the gap between the two quantities cannot be very large; this is an instance of approximate duality between multicommodity flows and cuts.

Theorem 7.2 (Approximate duality, edge version) For every n-vertex graph G we have

$$
\operatorname{espars}(G)=O\left(\frac{\log n}{\operatorname{econg}(G)}\right) .
$$

Although we won't really need this particular theorem, the proof can serve as an introduction to things we will actually use, and we present it in Section 9 .

Exercise 7.3 (Edge sparsity and balanced edge cut) Let $\beta>0$ and let $G$ be a graph on $n$ vertices such that espars $(H) \leq \beta$ for every induced subgraph $H$ of $G$ on at least $\frac{2}{3} n$ vertices. Show that $G$ has a balanced edge cut $(A, V \backslash A)$ with $|E(A, V \backslash A)| \leq \beta n^{2}$, where balanced means that $\frac{1}{3} n \leq|A| \leq \frac{2}{3} n$.

Vertex notions. For the proof of the separator theorem for string graphs, we will need vertex analogs of the "edge" notions and results just discussed.

For a flow $\varphi$ in $G$ we introduce the vertex congestion

$$
\operatorname{vcong}(\varphi):=\max _{v \in V} \operatorname{vcong}(v), \text { where } \operatorname{vcong}(v):=\sum_{P \in \mathcal{P}: v \in P} \frac{1}{2} \varphi(P),
$$

and the vertex congestion of $G$ is $\operatorname{vcong}(G):=\min _{\varphi} \operatorname{vcong}(\varphi)$, where the minimum is over all $\varphi$ with unit flow between every two vertices. The $\frac{1}{2}$ in the above formula should be interpreted as 1 if $v$ is an inner vertex of the path $P$, and as $\frac{1}{2}$ if $v$ is one of the end-vertices of $P$. We thus think of the flow along $P$ as incurring congestion $\frac{1}{2} \varphi(P)$ when entering a vertex and congestion $\frac{1}{2} \varphi(P)$ when leaving it. (This convention is a bit of a nuisance in the definition of vertex congestion, but later on, it will pay off when we pass to a "dual" notion.)

By a vertex cut in $G$ we mean a partition $(A, B, S)$ of $V$ into three disjoint subsets such that $A \neq \emptyset \neq B$ and there are no edges between $A$ and $B$ (this is

\footnotetext{
${ }^{3}$ What we call edge sparsity is often called just sparsity. This quantity is also closely related to the Cheeger constant, or edge expansion, of $G$, which is defined as $\min _{A \subseteq V: 1 \leq|A| \leq|V| / 2}(|E(A, V \backslash A)| /|A|)$.
} 
like in the definition of a separator, except that we do not require the sizes of $A$ and $B$ to be roughly the same).

If $\varphi$ sends unit flow between every pair of vertices, then the flows between $A$ and $B$ contribute a total flow of $|A| \cdot|B|$ through $S$, and moreover, from each vertex of $S$ we have a flow of $n-1$ to the remaining vertices. Thus the total congestion of the vertices in $S$ is at least $|A| \cdot|B|+\frac{1}{2}|S|(n-1)$. Losing a constant factor (and using $n \geq 2$ ), we bound this somewhat unwieldy expression from below by $\frac{1}{4}|A| \cdot|B|+\frac{1}{4}|S| n=\frac{1}{4}|A \cup S| \cdot|B \cup S|$.

This suggests to define the sparsity of a vertex cut $(A, B, S)$ as

$$
\operatorname{vspars}(A, B, S):=\frac{|S|}{|A \cup S| \cdot|B \cup S|},
$$

and the vertex sparsity of $G$ is $\operatorname{vspars}(G):=\min _{(A, B, S)} \operatorname{vspars}(A, B, S)$, where the minimum is over all vertex cuts.

By the considerations above, we have vcong $(G) \geq 1 /(4 \operatorname{vspars}(G))$. We will need the following analog of Theorem 7.2 .

Theorem 7.4 (Approximate duality, vertex version) For every connected $n$-vertex graph $G$ we have

$$
\operatorname{vspars}(G)=O\left(\frac{\log n}{\operatorname{vcong}(G)}\right)
$$

The proof is deferred to Section 11 .

Exercise 7.5 (Vertex sparsity and separators) Let $\alpha>0$ and let $G$ be a graph on $n$ vertices such that $\operatorname{vspars}(H) \leq \alpha$ for every induced subgraph $H$ of $G$ on at least $\frac{2}{3} n$ vertices. Show that $G$ has a separator of size at most $\alpha n^{2}$.

String graphs have large vertex congestion. The last ingredient in the proof of the separator theorem for string graphs is the following result about string graphs.

Proposition 7.6 For every connected string graph $G$ with $n$ vertices and $m$ edges, we have

$$
\frac{1}{\operatorname{vcong}(G)}=O\left(\frac{\sqrt{m}}{n^{2}}\right)
$$

This is the only specific property of string graphs used in the proof of the separator theorem. The next section is devoted to the proof of this proposition. Proof of the separator theorem for string graphs (Theorem 5.4). Let $G$ be a string graph with $n$ vertices and $m \geq n$ edges. We have $1 / \operatorname{vcong}(G)=$ $O\left(\sqrt{m} / n^{2}\right)$ by Proposition 7.6. By approximate duality (Theorem 7.4), we have $\operatorname{vspars}(G)=O\left((\log n) \sqrt{m} / n^{2}\right)$, and so $G$ has a separator of size $O(\sqrt{m} \log n)$ according to Exercise 7.5 .

Exercise 7.7 Let $G$ be a string graph with $m$ edges whose maximum degree is bounded by a constant $\Delta$. Derive from Theorem 7.2 (the edge version of the approximate duality) and from Proposition 7.6 that $G$ has a separator of size $O(\sqrt{m} \log m)$, where the implicit constant may depend on $\Delta$. 


\section{String graphs have large vertex congestion}

The strategy of the proof of Proposition 7.6 is this: Given a string representation of an $n$-vertex graph $G$ and a multicommodity flow in $G$ with a small vertex congestion, we will construct a drawing of $K_{n}$ in which only a small number of edge pairs cross. This will contradict the following result:

Lemma 8.1 For $n \geq 5, \operatorname{pcr}\left(K_{n}\right)=\Omega\left(n^{4}\right)$.

The proof of this lemma relies on the following fact.

Fact 8.2 In every plane drawing of $K_{5}$, some two independent edges intersect, where independent means that the edges do not share a vertex.

This fact is a consequence of the Hanani-Tutte theorem mentioned above Proposition 6.3. although that theorem is somewhat too big a hammer for this purpose. But proving the fact rigorously is harder than it may seem, even if we assume nonplanarity of $K_{5}$ as known (although a rigorous proof of the nonplanarity is almost never included in graph theory courses).

Exercise 8.3 Find a mistake in the following "proof" of Fact 8.2: Consider a (standard) drawing of $K_{5}$. If two independent edges cross, we are done, and otherwise, some two edges sharing a vertex cross. But such crossings can be removed by the following transformation

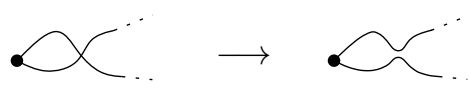

and so eventually we reach a plane drawing - a contradiction.

Proof of Lemma 8.1. By Fact 8.2, in every drawing of $K_{n}$, every 5-tuple of vertices induces a pair of independent edges that cross. A given pair of independent crossing edges determines 4 vertices of the 5-tuple, and so the number of 5 -tuples inducing this particular pair of edges is at most $n-4$. So $\operatorname{pcr}\left(K_{n}\right) \geq\left(\begin{array}{l}n \\ 5\end{array}\right) /(n-4)=\Omega\left(n^{4}\right)$.

We remark that Lemma 8.1 also follows from a generally useful result, the crossing lemma of Ajtai et al. ACNS82 and Leighton [Lei84, which asserts that every graph with $n$ vertices and $m \geq 4 n$ edges has crossing number $\Omega\left(m^{3} / n^{2}\right)$. We actually need a version of the lemma for the pair-crossing number, which holds with the same bound, as was observed in Pach and Tóth [PT00, Thm. 3] $!^{4}$ This proof does not avoid Fact 8.2 it actually relies on a generalization of it.

Proof of Proposition 7.6. Let $G=(V, E)$, and let $\left(\gamma_{v}: v \in V\right)$ be a string representation of $G$. We are going to produce a drawing of the complete graph $K_{V}$ on the vertex set $V$.

\footnotetext{
${ }^{4}$ The argument in their proof is not quite correct, but the problem is rectified in Remark 2 in Section 3 of PT00.
} 
We draw each vertex $v \in V$ as a point $p_{v} \in \gamma_{v}$, in such a way that all the $p_{v}$ are distinct.

For every edge $\{u, v\} \in\left(\begin{array}{c}V \\ 2\end{array}\right)$ of the complete graph, we pick a path $P_{u v}$ from $\mathcal{P}_{u v}$, in a way to be specified later. Let us enumerate the vertices along $P_{u v}$ as $v_{0}=u, v_{1}, v_{2}, \ldots, v_{k}=v$. Then we draw the edge $\{u, v\}$ of $K_{V}$ in the following manner: we start at $p_{u}$, follow $\gamma_{u}$ until some (arbitrarily chosen) intersection with $\gamma_{v_{1}}$, then we follow $\gamma_{v_{1}}$ until some intersection with $\gamma_{v_{2}}$, etc., until we reach $\gamma_{v}$ and $p_{v}$ on it.

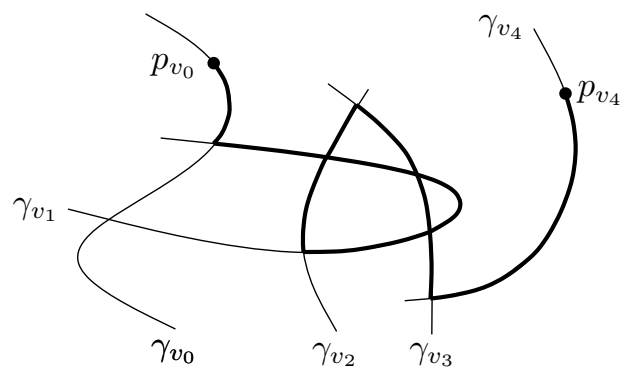

In this way we typically do not get a standard drawing, since edges may share segments, have self-intersections and triple points, and they may pass through vertices. However, we can obtain a standard drawing by shortcutting loops and a small perturbation of the edges, in such a way that no new intersecting pairs of edges are created. Hence, by Lemma 8.1, there are $\Omega\left(n^{4}\right)$ intersecting pairs of edges in the original drawing as well. We are going to estimate the number of intersecting pairs in a different way.

We note that the drawings of two edges $\{u, v\}$ and $\left\{u^{\prime}, v^{\prime}\right\}$ cannot intersect unless there are vertices $w \in P_{u v}$ and $w^{\prime} \in P_{u^{\prime} v^{\prime}}$ such that $\gamma_{w} \cap \gamma_{w^{\prime}} \neq \emptyset$, i.e., $\left\{w, w^{\prime}\right\} \in E(G)$ or $w=w^{\prime}$. Let us write this latter condition as $P_{u v} \sim P_{u^{\prime} v^{\prime}}$.

How do we select the paths $P_{u v}$ ? For this, we consider a flow $\varphi$ for which $\operatorname{vcong}(G)$ is attained. Since there is a unit flow between every pair of vertices $\{u, v\}$, the values of $\varphi(P)$ define a probability distribution on $\mathcal{P}_{u v}$. We choose $P_{u v} \in \mathcal{P}_{u v}$ from this distribution at random, the choices independent for different $\{u, v\}$.

The number $X$ of intersecting pairs of edges in this drawing is a random variable, and we bound from above its expectation. First we note that

$$
\begin{gathered}
\operatorname{Prob}\left[\{u, v\} \text { and }\left\{u^{\prime}, v^{\prime}\right\} \text { intersect }\right] \leq \operatorname{Prob}\left[P_{u v} \sim P_{u^{\prime} v^{\prime}}\right] \\
=\sum_{P \in \mathcal{P}_{u v}, P^{\prime} \in \mathcal{P}_{u^{\prime} v^{\prime}}, P \sim P^{\prime}} \operatorname{Prob}\left[P_{u v}=P \text { and } P_{u^{\prime} v^{\prime}}=P^{\prime}\right] \\
=\sum_{P \in \mathcal{P}_{u v}, P^{\prime} \in \mathcal{P}_{u^{\prime} v^{\prime}}, P \sim P^{\prime}} \varphi(P) \varphi\left(P^{\prime}\right)
\end{gathered}
$$


(for the last equality we have used independence). Therefore

$$
\begin{aligned}
\mathbf{E}[X] & =\sum_{\left\{\{u, v\},\left\{u^{\prime}, v^{\prime}\right\}\right\} \in\left(\begin{array}{c}
\left(\begin{array}{c}
V \\
2
\end{array}\right) \\
2
\end{array}\right)} \operatorname{Prob}\left[\{u, v\} \text { and }\left\{u^{\prime}, v^{\prime}\right\} \text { intersect }\right] \\
\leq & \sum_{\{u, v\},\left\{u^{\prime}, v^{\prime}\right\}} \sum_{P \in \mathcal{P}_{u v}, P^{\prime} \in \mathcal{P}_{u^{\prime} v^{\prime}}, P \sim P^{\prime}} \varphi(P) \varphi\left(P^{\prime}\right) \\
& \sum_{\left\{w, w^{\prime}\right\} \in E \text { or } w=w^{\prime}} \varphi(P) \varphi\left(P^{\prime}\right) \\
& \sum_{P, P^{\prime} \in \mathcal{P}, w \in P, w^{\prime} \in P^{\prime}}\left(\sum_{\left\{w, w^{\prime}\right\} \in E \text { or } w=w^{\prime}} \varphi(P)\right)\left(\sum_{P \in \mathcal{P}, w \in P} \varphi\left(P^{\prime}\right)\right) .
\end{aligned}
$$

The first sum in parentheses is at most $2 \operatorname{vcong}(w)$, and the second one at most $2 \operatorname{vcong}\left(w^{\prime}\right)$; the 2 is needed because of the paths $P$ for which $w$ is an endvertex. The number of terms in the outer sum is $|E|+n \leq 2 m$. Altogether we get $\mathbf{E}[X] \leq 8 m \operatorname{vcong}(G)^{2}$.

Since, on the other hand, we always have $X=\Omega\left(n^{4}\right)$, we obtain $1 / \operatorname{vcong}(G)=$ $O\left(\sqrt{m} / n^{2}\right)$ as claimed.

\section{Flows, cuts, and metrics: the edge case}

Here we prove the edge version of the approximate flow/cut duality, Theorem 7.2. We essentially follow an argument of Linial, London, and Rabinovich [LLR95.

Dualizing the linear program. The first step of the proof can be concisely expressed as follows: express econg $(G)$ by a linear program, dualize it, and see what the dual means 5

It is slightly nicer to work with $\frac{1}{\operatorname{econg}(G)}$, which can be expressed as the maximum $t$ such that there is a flow $\varphi$ with edge congestion at most 1 that sends at least $t$ between every pair of vertices. The resulting linear program has variables $t \geq 0$ and $\varphi(P), P \in \mathcal{P}$, and it looks like this:

$$
\begin{aligned}
\max \{t \geq 0: & \varphi(P) \geq 0 \text { for all } P \in \mathcal{P} \\
& \sum_{P: e \in P} \varphi(P) \leq 1 \text { for all } e \in E, \\
& \left.\sum_{P \in \mathcal{P}_{u v}} \varphi(P) \geq t \text { for all }\{u, v\} \in\left(\begin{array}{c}
V \\
2
\end{array}\right)\right\} .
\end{aligned}
$$

\footnotetext{
${ }^{5}$ We assume that the reader has heard about linear programming and the duality theorem in it; if not, we recommend consulting a suitable source. Here is a very brief summary: A linear program is the computational problem of maximizing (or minimizing) a linear function over the intersection of finitely many half-spaces in $\mathbb{R}^{n}$ (i.e., a convex polyhedron). Every linear program can be converted to a standard form: $\max _{\boldsymbol{x} \in P} \boldsymbol{c}^{T} \boldsymbol{x}$ with $P=\{\boldsymbol{x} \in$ $\left.\mathbb{R}^{n}: A \boldsymbol{x} \leq \boldsymbol{b}, \boldsymbol{x} \geq \mathbf{0}\right\}$, where $\boldsymbol{c} \in \mathbb{R}^{n}, \boldsymbol{b} \in \mathbb{R}^{m}, A$ is an $m \times n$ matrix, and the inequalities between vectors are meant componentwise. The dual of this linear program is $\min _{\boldsymbol{y} \in D} \boldsymbol{b}^{T} \boldsymbol{y}$, $D=\left\{\boldsymbol{y} \in \mathbb{R}^{m}, A^{T} \boldsymbol{y} \geq \boldsymbol{c}, \boldsymbol{y} \geq \mathbf{0}\right\}$, and the duality theorem of linear programming asserts that if $P \neq \emptyset \neq D$, then $\min _{\boldsymbol{x} \in P} \boldsymbol{c}^{T} \boldsymbol{x}=\max _{\boldsymbol{y} \in D} \boldsymbol{b}^{T} \boldsymbol{y}$.
} 
The variables of the dual linear program are $x_{e}, e \in E$, corresponding to the constraints [1], and $y_{u v},\{u, v\} \in\left(\begin{array}{l}V \\ 2\end{array}\right)$, corresponding to the constraints (2). The dual reads

$$
\begin{aligned}
\min \left\{\sum_{e \in E} x_{e}:\right. & x_{e}, y_{u v} \geq 0 \\
& \sum_{e \in P} x_{e} \geq y_{u v} \text { for every } P \in \mathcal{P}_{u v},\{u, v\} \in\left(\begin{array}{c}
V \\
2
\end{array}\right), \\
& \left.\sum_{\{u, v\} \in\left(\begin{array}{c}
V \\
2
\end{array}\right)} y_{u v} \geq 1\right\}
\end{aligned}
$$

and its value also equals $\frac{1}{\operatorname{econg}(G)}$ by the duality theorem. (Checking this claim carefully takes some work, and we expect only the most diligent readers to verify it - the others may simply take it for granted, since the linear programming duality is a side-topic for us.)

Fortunately, the dual linear program has a nice interpretation. We think of the variables $x_{e}$ as edge weights, and then the constraints (3) say that $y_{u v}$ is at most the sum of weights along every $u-v$ path. From this it is easy to see that in an optimal solution of the dual linear program, each $y_{u v}$ is the length of a shortest $u-v$ path under the edge weights given by the $x_{e}$. When the $y_{u v}$ are given in this way, we may also assume that for every edge $e=\{u, v\} \in E$, we have $x_{e}=y_{u v}$ : Indeed, if $x_{e}>y_{u v}$, then there is a shortcut between $u$ and $v$ bypassing the edge $e$, i.e., a $u-v$ path of length $y_{u v}$. So if we decrease $x_{e}$ to the value $y_{u v}$, the length of a shortest path between every two vertices remains unchanged and thus no inequality in the linear program is violated, while $\sum_{e \in E} x_{e}$ decreases.

Thus, if we write $d_{w}$ for the shortest-path (pseudo)metric ${ }^{6}$ induced on $V$ by an edge weight function $w: E \rightarrow[0, \infty)$, we can express the conclusion of the dualization step as

$$
\frac{1}{\operatorname{econg}(G)}=\min \left\{\frac{\sum_{\{u, v\} \in E} d_{w}(u, v)}{\sum_{\{u, v\} \in\left(\begin{array}{c}
V \\
2
\end{array}\right)} d_{w}(u, v)}: w: E \rightarrow[0, \infty), w \neq \equiv 0\right\} .
$$

Here $w \not \equiv 0$ means that $w$ is not identically 0 ; note that we replaced the constraint (4), requiring the sum of all distances under $d_{w}$ to be at least 1 , by dividing the minimized function by the sum of all distances.

Cut metrics and line metrics. To make further progress, we will investigate the minimum of the same ratio as in (5), but over different classes of metrics.

A cut metric on a set $V$ is a pseudometrid 7 given by $c(u, v)=|f(u)-f(v)|$ for some function $f: V \rightarrow\{0,1\}$.

By comparing the definitions, we can express the edge sparsity of a graph

\footnotetext{
${ }^{6}$ We recall that a metric on a set $V$ is a mapping $d: V \times V \rightarrow[0, \infty)$ satisfying (i) $d(u, v)=d(v, u)$ for all $u, v$; (ii) $d(u, u)=0$ for all $u$; (iii) $d(u, v)>0$ whenever $u \neq v$; and (iv) $d(u, v) \leq d(u, x)+d(x, v)$ for all $u, v, x \in V$ (triangle inequality). A pseudometric satisfies the same axioms except possibly for (iii).

${ }^{7}$ Cut metric is really a misnomer, since a cut metric is almost never a metric; we should speak of a cut pseudometric, but we conform to the usage in the literature. A similar remark applies to line metrics considered below.
} 
as

$$
\operatorname{espars}(G)=\min \left\{\frac{\sum_{\{u, v\} \in E} c(u, v)}{\sum_{\{u, v\} \in\left(\begin{array}{c}
V \\
2
\end{array}\right)} c(u, v)}: c \text { a cut metric on } V, c \not \equiv 0\right\}
$$

(please check).

Next, it turns out that we can replace cut metrics by line metrics in (6) and the minimum stays the same. Here a line metric is a pseudometric $\ell$ such that $\ell(u, v)=|f(u)-f(v)|$ for some function $f: V \rightarrow \mathbb{R}$. We leave the proof as an instructive exercise.

Exercise 9.1 Show that the minimum in

$$
\min \left\{\frac{\sum_{\{u, v\} \in E} \ell(u, v)}{\sum_{\{u, v\} \in\left(\begin{array}{c}
V \\
2
\end{array}\right)} \ell(u, v)}: \ell \text { a line metric on } V, \ell \neq \equiv 0\right\}
$$

is attained by a cut metric, and hence it also equals espars $(G)$. (Hint: show that if the function $f$ defining a line metric $\ell$ attains at least three distinct values, then some value can be eliminated.)

A key result that allows us to compare the minimum (5) over all shortestpath metrics with the minimum (7) over all line metric follows from the work of Bourgain [Bou85]. His main theorem was formulated differently, but his proof immediately yields the following formulation, which is the most convenient for our purposes.

Theorem 9.2 Let $V$ be an n-point set. For every (pseudo)metric d on $V$ there exists a line metric $\ell$ on $V$ satisfying

(i) ( $\ell$ is below $d) \ell(u, v) \leq d(u, v)$ for all $u, v \in V$, and

(ii) (the average distance not decreased too much)

$$
\sum_{\{u, v\} \in\left(\begin{array}{c}
V \\
2
\end{array}\right)} \ell(u, v) \geq \frac{c}{\log n} \sum_{\{u, v\} \in\left(\begin{array}{c}
V \\
2
\end{array}\right)} d(u, v),
$$

for a constant $c>0$.

For completeness, we demonstrate the main idea of the proof in Section 10 below.

Proof of Theorem 7.2, Let $d^{*}$ be a shortest-path metric attaining the minimum in the expression (5) for $\frac{1}{\operatorname{econg}(G)}$. We apply Theorem 9.2 with $d=d^{*}$ and obtain a line metric $\ell^{*}$ satisfying (i), (ii) in the theorem. Then

$$
\begin{aligned}
\frac{1}{\operatorname{econg}(G)} & =\frac{\sum_{\{u, v\} \in E} d^{*}(u, v)}{\sum_{\{u, v\} \in\left(\begin{array}{c}
V \\
2
\end{array}\right)} d^{*}(u, v)} \geq \frac{c}{\log n} \cdot \frac{\sum_{\{u, v\} \in E} \ell^{*}(u, v)}{\sum_{\{u, v\} \in\left(\begin{array}{c}
V \\
2
\end{array}\right)} \ell^{*}(u, v)} \\
& \geq \frac{c}{\log n} \cdot \min \left\{\frac{\sum_{\{u, v\} \in E} \ell(u, v)}{\sum_{\{u, v\} \in\left(\begin{array}{c}
V \\
2
\end{array}\right)} \ell(u, v)}: \ell \text { a line metric on } V, \ell \neq \equiv 0\right\} \\
& =\frac{c}{\log n} \cdot \operatorname{espars}(G) .
\end{aligned}
$$




\section{Proof of a weaker version of Bourgain's theorem}

Here we prove a version of Theorem 9.2 with $\log n$ replaced by $\log ^{2} n$; this weakening makes the proof simpler, while preserving the main ideas.

Providing a line metric $\ell$ satisfying condition (i), $\ell \leq d$, is equivalent to providing a function $f: V \rightarrow \mathbb{R}$ that is 1-Lipschitz, i.e., satisfies $|f(u)-f(v)| \leq$ $d(u, v)$ for all $u, v \in V$.

A suitable $f$ is chosen at random, in the following steps.

1. Let $k$ be the smallest integer with $2^{k} \geq n$, i.e., $k=\left\lceil\log _{2} n\right\rceil$. Choose an index $j \in\{0,1, \ldots, k\}$ uniformly at random, and set $p:=2^{-j}$.

2. Choose a random subset $A \subseteq V$, where each point $v \in V$ is included in $A$ independently with probability $p$.

3. Define $f$ by $f(u):=d(u, A)=\min _{a \in A} d(u, a)$.

A nice thing about this way of choosing $f$ is that it is 1-Lipschitz for every $A \subseteq V$, as can be easily checked using the triangle inequality. So it remains to show that, with positive probability, the line metric induced by $f$ satisfies a weaker version of condition (ii), i.e., that it does not decrease the average distance too much.

We will actually prove that for every $u, v \in V, u \neq v$,

$$
\operatorname{Prob}\left[|f(u)-f(v)| \geq \frac{c_{0}}{\log n} \cdot d(u, v)\right] \geq \frac{c_{0}}{\log n},
$$

where the probability is with respect to the random choice of $f$ as above, and $c_{0}>0$ is a suitable constant. Assuming (8), passing to expectation, and summing over $\{u, v\} \in\left(\begin{array}{c}V \\ 2\end{array}\right)$, we arrive at

$$
\mathbf{E}\left[\sum_{\{u, v\} \in\left(\begin{array}{c}
V \\
2
\end{array}\right)}|f(u)-f(v)|\right] \geq \frac{c_{0}^{2}}{\log ^{2} n} \sum_{\{u, v\} \in\left(\begin{array}{c}
V \\
2
\end{array}\right)} d(u, v),
$$

and hence at least one $f$ satisfies (ii) with $\log ^{2} n$ instead of $\log n$.

So we fix $u, v$ and we aim at proving (8). Let us set $\Delta:=d(u, v) /(2 k-1)$. We have $|f(u)-f(v)|=|d(u, A)-d(v, A)|$, and the latter expression is at least $\Delta$ provided that, for some $r \geq 0$, the set $A$ intersects the (closed) $r$-ball around $u$ and avoids the (open) $(r+\Delta)$ ball around $v$, or the other way round.

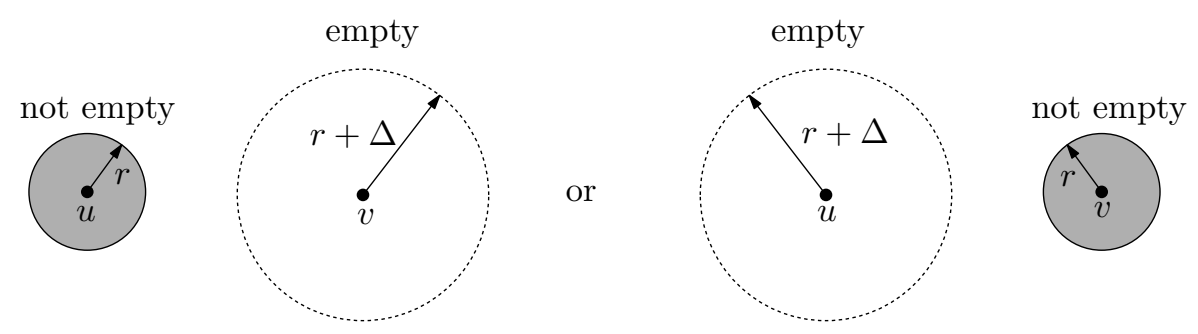

In order for this event to have a non-negligible probability, we need that the number of points in the bigger balls is not much larger than in the smaller ball. The trick for achieving this is to consider a system of balls as in the next picture: 


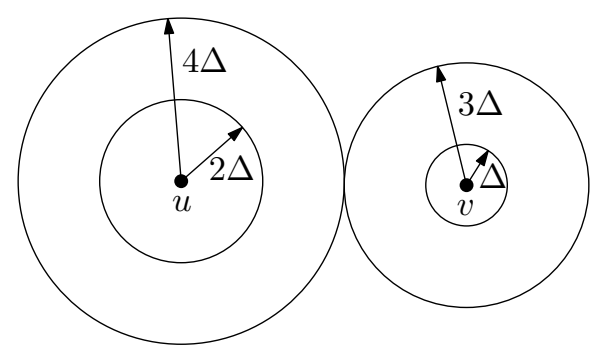

The picture is for $k=4$. In general, $B_{i}$ is the closed ball of radius $i \Delta, i=$ $0,1, \ldots, k$, centered at $u$ for even $i$ and at $v$ for odd $i$. Let $B_{i}^{\circ}$ denote the corresponding open ball (all points at distance strictly smaller than $i \Delta$ from the center).

Let $n_{i}$ be the number of points in $B_{i}$. We claim that $n_{i+1} / n_{i} \leq 2$ for some $i \in\{0,1, \ldots, k-1\}$; indeed, if not, then $\left|B_{k}\right|>2^{k} \geq n-$ a contradiction.

We fix such an $i$, and we also fix $j_{0}$ such that $n_{i}$ is approximately $2^{j_{0}}$; more precisely, $2^{j_{0}} \leq n_{i}<2^{j_{0}+1}$.

Let $p=2^{-j_{0}}$, and let us pick a random $A$ as in the second step of the choice of $f$ with this value of $p$. By a simple calculation, which we leave as an exercise, there is a constant $c_{1}>0$ such that

$$
\operatorname{Prob}\left[A \cap B_{i} \neq \emptyset \text { and } A \cap B_{i+1}^{\circ}=\emptyset\right] \geq c_{1} .
$$

Exercise 10.1 Let $X, Y$ be disjoint sets, and let $A \subseteq X \cup Y$ be a random subset, where each point of $X \cup Y$ is included in $A$ with probability $p$, independent of all other points, with $0<p \leq \frac{1}{2}$. Assuming $\frac{1}{2 p} \leq|X|,|Y| \leq \frac{2}{p}$, show that $\operatorname{Prob}[A \cap X \neq \emptyset$ and $A \cap Y=\emptyset] \geq c_{1}$ for a constant $c_{1}>0$.

Now (8) follows easily: given $u, v$, the probability of choosing $j=j_{0}$ is $\frac{1}{k+1}=$ $\Omega\left(\frac{1}{\log n}\right)$, and conditioned on this choice, we have $\operatorname{Prob}[|f(u)-f(v)| \geq \Delta] \geq c_{1}$. This concludes the proof of the weaker version of Theorem 9.2.

\section{Flows, cuts, and metrics: the vertex case}

Here we prove Theorem 7.4, the vertex case of the approximate duality, and this will also conclude the quest for the proof of the separator theorem for string graphs. Initially we proceed in a way similar to the edge case from Section 9 . but the last step is more demanding and uses a nice method for producing sparse vertex cuts algorithmically.

Dualization again. As before, we write $\frac{1}{\operatorname{vcong}(G)}$ as a linear program and dualize it. The linear program differs from the one for $\frac{1}{\operatorname{econg}(G)}$ only in the second line:

$$
\begin{aligned}
\max \{t \geq 0: & \varphi(P) \geq 0 \text { for all } P \in \mathcal{P} \\
& \sum_{P: v \in P} \frac{1}{2} \varphi(P) \leq 1 \text { for all } v \in V, \\
& \left.\sum_{P \in \mathcal{P}_{u v}} \varphi(P) \geq t \text { for all }\{u, v\} \in\left(\begin{array}{c}
V \\
2
\end{array}\right)\right\} ;
\end{aligned}
$$


here the meaning of $\frac{1}{2}$ is as in the definition of $\operatorname{vcong}(G)$ in Section 7. In the dual, we have variables $y_{u v}$ indexed by pairs of vertices and $z_{v}$ indexed by vertices, and it reads

$$
\begin{aligned}
\min \left\{\sum_{z \in V} z_{v}: \quad\right. & z_{v}, y_{u v} \geq 0, \\
& \sum_{v \in P} \frac{1}{2} z_{v} \geq y_{u v} \text { for every } P \in \mathcal{P}_{u v},\{u, v\} \in\left(\begin{array}{c}
V \\
2
\end{array}\right), \\
& \left.\sum_{\{u, v\} \in\left(\begin{array}{c}
V \\
2
\end{array}\right)} y_{u v} \geq 1\right\} .
\end{aligned}
$$

This, too, can be interpreted using a metric on $G$. This time we have a function $s: V \rightarrow[0, \infty)$ assigning weights to vertices. Let us define the derived weight of an edge $e=\{u, v\}$ by $w(e):=\frac{1}{2}(s(u)+s(v))$ and denote the corresponding shortest-path metric by $d_{s}$. Then, in a way very similar to the edge case, one can see that

$$
\frac{1}{\operatorname{vcong}(G)}=\min \left\{\frac{\sum_{v \in V} s(v)}{\sum_{\{u, v\} \in\left(\begin{array}{c}
V \\
2
\end{array}\right)} d_{s}(u, v)}: s: V \rightarrow[0, \infty), s \not \equiv 0\right\} .
$$

Here the convention with $\frac{1}{2}$ for the vertex congestion pays off- the dual has a nice interpretation in terms of shortest-path metrics.

Let $s^{*}$ be a weight function for which the minimum in $(13)$ is attained. Applying Bourgain's theorem (Theorem 9.2) to the metric $d_{s^{*}}$ yields a function $f^{*}: V \rightarrow \mathbb{R}$ that is 1 -Lipschitz w.r.t. $d_{s^{*}}$ and satisfies

$$
\frac{\sum_{v \in V} s(v)}{\sum_{\{u, v\} \in\left(\begin{array}{c}
V \\
2
\end{array}\right)}\left|f^{*}(u)-f^{*}(v)\right|}=O\left(\frac{\log n}{\operatorname{vcong}(G)}\right) .
$$

The following theorem of Feige, Hajiaghayi, and Lee [FHL08] then shows how such an $f^{*}$ can be used to produce sparse vertex cuts in $G$. This is the last step in the proof of Theorem 7.4 .

Theorem 11.1 Let $G$ be a graph, $s: V \rightarrow[0, \infty)$ a weight function on the vertices, $d_{s}$ the corresponding metric, and let $f: V \rightarrow \mathbb{R}$ be a non-constant 1-Lipschitz function w.r.t. $d_{s}$. Then

$$
\operatorname{vspars}(G) \leq \frac{\sum_{v \in V} s(v)}{\sum_{\{u, v\} \in\left(\begin{array}{c}
V \\
2
\end{array}\right)}|f(u)-f(v)|} .
$$

Proof. The proof actually provides a polynomial-time algorithm for finding a vertex cut with sparsity bounded as in the theorem.

Let us number the vertices of $G$ so that $f\left(v_{1}\right) \leq f\left(v_{2}\right) \leq \cdots \leq f\left(v_{n}\right)$. For every $i=1,2, \ldots, n-1$, we are going to find a vertex cut $\left(A_{i}, B_{i}, S_{i}\right)$, and show that one of these will do.

To this end, given $i$, we form an auxiliary graph $G_{i}^{+}$by adding new vertices $x$ and $y$ to $G$, connecting $x$ to $v_{1}$ through $v_{i}$, and $y$ to $v_{i+1}$ through $v_{n}$.

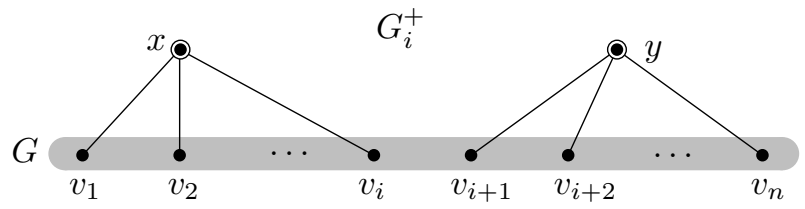


We let $S_{i} \subseteq V$ be a minimum cut in $G_{i}^{+}$separating $x$ from $y$ (which can be found using a max-flow algorithm, for example). Let $A_{i}:=\left\{v_{1}, \ldots, v_{i}\right\} \backslash S_{i}$ and $B_{i}:=\left\{v_{i+1}, \ldots, v_{n}\right\} \backslash S_{i}$, and let

$$
\alpha:=\min _{i} \operatorname{vspars}\left(A_{i}, B_{i}, S_{i}\right)=\min _{i} \frac{\left|S_{i}\right|}{\left|A_{i} \cup S_{i}\right| \cdot\left|B_{i} \cup S_{i}\right|} .
$$

Since $\left\{v_{1}, \ldots, v_{i}\right\} \subseteq A_{i} \cup S_{i}$, we have $\left|A_{i} \cup S_{i}\right| \geq i$, and similarly $\left|B_{i} \cup S_{i}\right| \geq n-i$. Thus, for every $i$ we have

$$
\left|S_{i}\right| \geq \alpha i(n-i)
$$

In order to prove the theorem, we want to derive

$$
\alpha \sum_{\{u, v\} \in\left(\begin{array}{c}
V \\
2
\end{array}\right)}|f(u)-f(v)| \leq \sum_{v \in V} s(v) .
$$

Setting $\varepsilon_{i}=f\left(v_{i+1}\right)-f\left(v_{i}\right) \geq 0$, we can rearrange the left-hand side: $\alpha \sum_{i<j}\left(f\left(v_{j}\right)-\right.$ $\left.f\left(v_{i}\right)\right)=\alpha \sum_{i=1}^{n-1} i(n-i) \varepsilon_{i}$ (we just look how many times the segment between $f\left(v_{i}\right)$ and $f\left(v_{i+1}\right)$ is counted). Then, substituting from (14), we finally bound the left-hand side of $(15)$ by $\sum_{i=1}^{n-1} \varepsilon_{i}\left|S_{i}\right|$. It remains to prove

$$
\sum_{i=1}^{n-1} \varepsilon_{i}\left|S_{i}\right| \leq \sum_{v \in V} s(v),
$$

and this is the most ingenious part of the proof.

Roughly speaking, for every term $\varepsilon_{i}\left|S_{i}\right|$, we want to find vertices of sufficient total weight sufficiently close to the interval $\left[f\left(v_{i}\right), f\left(v_{i+1}\right)\right]$. We use Menger's theorem, which guarantees that there are $\left|S_{i}\right|$ vertex-disjoint paths that have to "jump over" the interval $\left[f\left(v_{i}\right), f\left(v_{i+1}\right)\right]$.

More precisely, we express both sides of (16) as integrals. Namely, we write $\sum_{i=1}^{n-1} \varepsilon_{i}\left|S_{i}\right|=\int_{-\infty}^{\infty} g(z) \mathrm{d} z$, where $g$ is the function that equals $\left|S_{i}\right|$ on $\left[f\left(v_{i}\right), f\left(v_{i+1}\right)\right)$ and 0 elsewhere:

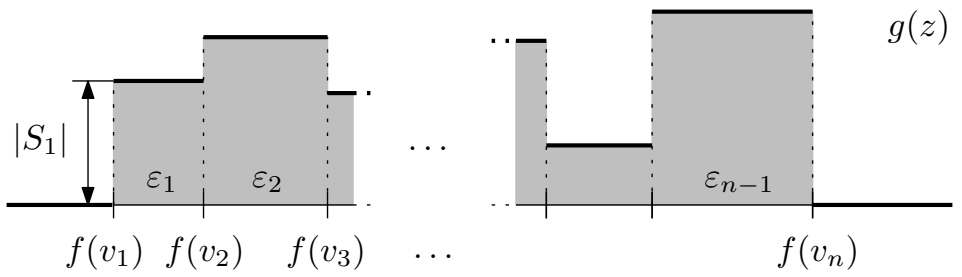

Similarly, $\sum_{v \in V} s(v)=\int_{-\infty}^{\infty} \sum_{i=1}^{n} h_{i}(z) \mathrm{d} z$, where $h_{i}$ is the function equal to 1 on $\left[f\left(v_{i}\right)-\frac{s\left(v_{i}\right)}{2}, f\left(v_{i}\right)+\frac{s\left(v_{i}\right)}{2}\right]$ and to 0 elsewhere:

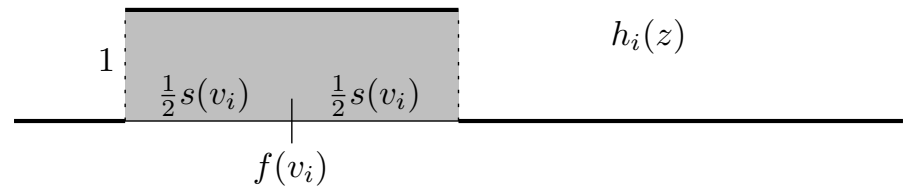

We claim that $g(z) \leq \sum_{i=1}^{n} h_{i}(z)$ for every $z \in \mathbb{R}$; this will imply (16).

Let $z \in\left[f\left(v_{i}\right), f\left(v_{i+1}\right)\right]$, and set $m=g(z)=\left|S_{i}\right|$. We want to show $\sum_{i=1}^{n} h_{i}(z) \geq m$, which means that we need to find $m$ distinct vertices $v$ such 
that $|f(v)-z| \leq \frac{s(v)}{2}$; let us call such $v$ the paying vertices since we can imagine that they pay for $g(z)$.

As announced, we use Menger's theorem, which tells us that, since $S_{i}$ is a minimum $x$ - $y$ cut in $G_{i}^{+}$, there are $m x-y$ paths $P_{1}, \ldots, P_{m}$ that are vertex-disjoint except for sharing the end-vertices $x$ and $y$. Each $P_{j}$ contains at least one edge $e_{j}=\left\{a_{j}, b_{j}\right\}$ with one endpoint among $v_{1}, \ldots, v_{i}$ and the other among $v_{i+1}, \ldots, v_{n}$. Hence $z \in\left[f\left(a_{j}\right), f\left(b_{j}\right)\right]$, and since $f$ is 1-Lipschitz, $\left|f\left(a_{j}\right)-f\left(b_{j}\right)\right| \leq d_{s}\left(a_{j}, b_{j}\right) \leq \frac{1}{2}\left(s\left(a_{j}\right)+s\left(b_{j}\right)\right)$. Thus, we have $\left|f\left(a_{j}\right)-z\right| \leq \frac{s\left(a_{j}\right)}{2}$ or $\left|f\left(b_{j}\right)-z\right| \leq \frac{s\left(b_{j}\right)}{2}$ (or both), and so $a_{j}$ or $b_{j}$ is a paying vertex. This gives the desired $m$ distinct paying vertices.

\section{References}

[ACNS82] Miklós Ajtai, Václav Chvátal, Monroe M. Newborn, and Endre Szemerédi. Crossing-free subgraphs. Ann. Discrete Math., 12:9-12, 1982 .

[ARV09] Sanjeev Arora, Satish Rao, and Umesh Vazirani. Expander flows, geometric embeddings and graph partitioning. J. ACM, 56(2):Art. $5,37,2009$.

[AST94] Noga Alon, Paul Seymour, and Robin Thomas. Planar separators. SIAM J. Discrete Math., 2(7):184-193, 1994.

[Ber95] Claude Berge. Who killed the Duke of Densmore? In Queneau, Raymond, Italo Calvino, et al., Oulipo Laboratory. Atlas Press, London, 1995 .

[Bou85] Jean Bourgain. On Lipschitz embedding of finite metric spaces in Hilbert space. Israel J. Math., 52:46-52, 1985.

[Cab13] Sergio Cabello. Hardness of approximation for crossing number. Discrete Comput. Geom., 49(2):348-358, 2013.

[CG09] Jérémie Chalopin and Daniel Gonçalves. Every planar graph is the intersection graph of segments in the plane. In Proc. 41st Annual ACM Symposium on Theory of Computing (STOC), pages 631-638, New York, NY, USA, 2009. ACM.

[CGO10] Jérémie Chalopin, Daniel Gonçalves, and Pascal Ochem. Planar graphs have 1-string representations. Discrete Comput. Geom., 43(3):626-647, 2010.

[Chu11] Julia Chuzhoy. An algorithm for the graph crossing number problem. In Proceedings of the 43rd annual ACM symposium on Theory of Computing (STOC), pages 303-312, 2011. Full version in arXiv:1012.0255. 
[CMS11] Julia Chuzhoy, Yury Makarychev, and Anastasios Sidiropoulos. On graph crossing number and edge planarization. In Proceedings of the Twenty-Second Annual ACM-SIAM Symposium on Discrete Algorithms, pages 1050-1069. SIAM, Philadelphia, PA, 2011.

[EGS03] Guy Even, Sudipto Guha, and Baruch Schieber. Improved approximations of crossings in graph drawings and VLSI layout areas. SIAM J. Comput., 32(1):231-252 (electronic), 2002/03.

[FHL08] Uriel Feige, Mohammad T. Hajiaghayi, and James R. Lee. Improved approximation algorithms for minimum weight vertex separators. SIAM Journal on Computing, 38(2):629-657, 2008.

[FP10] Jacob Fox and János Pach. A separator theorem for string graphs and its applications. Combinatorics, Probability 83 Computing, 19(3):371-390, 2010.

[FP14] Jacob Fox and János Pach. Applications of a new separator theorem for string graphs. Combinatorics, Probability and Computing, 23:6674, 2014. Preprint arXiv:1302.7228.

[Gro04] Martin Grohe. Computing crossing numbers in quadratic time. J. Comput. System Sci., 68(2):285-302, 2004.

[HLW06] Shlomo Hoory, Nathan Linial, and Avi Widgerson. Expander graphs and their applications. Bull. Am. Math. Soc., New Ser., 43(4):439$561,2006$.

[KM91] Jan Kratochvíl and Jiří Matoušek. String graphs requiring exponential representations. J. Combin. Theory Ser. B, 53(1):1-4, 1991.

[KR07] Ken-ichi Kawarabayashi and Buce Reed. Computing crossing number in linear time. In Proc. 39th Annual ACM Symposium on Theory of Computing (STOC), pages 382-390, 2007.

[Kra91] Jan Kratochvíl. String graphs. II: Recognizing string graphs is NPhard. J. Comb. Theory, Ser. B, 52(1):67-78, 1991.

[Lei84] Frank T. Leighton. New lower bound techniques for VLSI. Math. Systems Theory, 17:47-70, 1984.

[LLR95] Nathan Linial, Eran London, and Yuri Rabinovich. The geometry of graphs and some its algorithmic applications. Combinatorica, 15:215-245, 1995.

[LR99] Frank T. Leighton and Satish Rao. Multicommodity max-flow mincut theorems and their use in designing approximation algorithms. J. Assoc. Comput. Machin., 46:787-832, 1999.

[Mat14] Jiř́ Matoušek. Near-optimal separators in string graphs. Combinatorics, Probability and Computing, 23:135-139, 2014. 
[PA95] János Pach and Pankaj K. Agarwal. Combinatorial Geometry. John Wiley \& Sons, New York, NY, 1995.

[PSŠ08] Michael J. Pelsmajer, Marcus Schaefer, and Daniel Štefankovič. Odd crossing number and crossing number are not the same. Discrete Comput. Geom., 39(1-3):442-454, 2008.

[PT00] János Pach and Géza Tóth. Which crossing number is it anyway? J. Combin. Theory Ser. B, 80:225-246, 2000.

[Sch14] Marcus Schaefer. Hanani-Tutte and related results. In Geometry Intuitive, Discrete, and Convex (Bólyai Society Mathematical Studies, Vol. 24, I. Bárány et al. eds). Springer, Berlin, 2014.

[SŠ04] Marcus Schaefer and Daniel Štefankovič. Decidability of string graphs. J. Comput. Syst. Sci., 68:319-334, 2004. Preliminary version in Proc. 33rd Annual ACM Symposium on Theory of Computing, 2001.

[SSŠ03] Marcus Schaefer, Eric Sedgwick, and Daniel Štefankovič. Recognizing string graphs in NP. J. Comput. Syst. Sci., 67(2):365-380, 2003 .

[Tót12] Géza Tóth. A better bound for pair-crossing number. In J. Pach ed., Thirty Essays on Geometric Graph Theory, pages 563-567. Springer, Berlin, 2012. 